\title{
Fluid \& Gyrokinetic turbulence in open field-line, helical plasmas
}

\author{
M. Francisquez, ${ }^{1}$ T. N. Bernard, ${ }^{2,3}$ B. Zhu, ${ }^{4}$ A. Hakim, ${ }^{5}$ B. N. Rogers, ${ }^{6}$ and G. W. Hammett ${ }^{5}$ \\ ${ }^{1)}$ MIT Plasma Science and Fusion Center, Cambridge, MA 02139a) \\ ${ }^{2)}$ General Atomics, PO Box 85608, San Diego, CA 92186, USA \\ ${ }^{3)}$ Institute for Fusion Studies, University of Texas at Austin, Austin, TX 78712, USA \\ ${ }^{4)}$ Lawrence Livermore National Laboratory, Livermore, CA 94550, USA \\ 5) Princeton Plasma Physics Laboratory, Princeton, NJ 08543, USA \\ ${ }^{6)}$ Dartmouth College, Hanover, NH 03755, USA
}

(Dated: 11 August 2020)

\begin{abstract}
Two-fluid Braginskii codes have simulated open-field line turbulence for over a decade, and only recently has it become possible to study these systems with continuum gyrokinetic codes. This work presents a first-of-its-kind comparison between fluid and (long-wavelength) gyrokinetic models in open field-lines, using the GDB and Gkeyll codes to simulate interchange turbulence in the Helimak device at the University of Texas (T. N. Bernard, et. al., Phys. of Plasmas 26, 042301 (2019)). Partial agreement is attained in a number of diagnostic channels when the GDB sources and sheath boundary conditions (BCs) are selected carefully, especially the heat-flux BCs which can drastically alter the temperature. The radial profile of the fluctuation levels is qualitatively similar and quantitatively comparable on the low-field side, although statistics such as moments of the probability density function and the high-frequency spectrum show greater differences. This comparison indicates areas for future improvement in both simulations, such as sheath BCs, as well as improvements in GDB like particle conservation and spatially varying thermal conductivity, in order to achieve better fluid-gyrokinetic agreement and increase fidelity when simulating experiments.
\end{abstract}

\section{MOTIVATION AND OVERVIEW}

Helical magnetic field devices, such as the Helimak (University of Texas) and TORPEX (EPFL), provide a useful environment for refining our understanding of open field-line toroidal systems. These devices have important ingredients of tokamak scrape-off layer (SOL) turbulence: parallel transport onto sheath regions, turbulent cross-field transport, curvature and $\nabla B$ drifts, and interaction with plasma-facing materials, main chamber neutrals and radio-frequency (RF) sources. Numerous aspects of tokamak fusion plasma operation are highly dependent on the conditions in the SOL. The fusion performance of the core, for example, is thought to be directly dependent on the plasma temperature at the pedestal top ${ }^{1}$ which is dynamically affected by the properties of the SOL plasma. The SOL is the site of sometimes deleterious field-aligned motion of particles and heat towards the tiles of the vessel wall. These are complicated and, to some extent, mitigated by the cross-field transport spreading loads over a larger surface of the wall ${ }^{2}$. Cross-field transport is not always desirable however, since coherent structures ${ }^{3}$ and edge-localized modes ${ }^{4}$ can also impact the walls and cause sputtering, penetration of impurities and plasma cooling, all of which undermine the performance of the core. Understanding these processes, and gaining the ability to predict and optimize them, are highly desirable for designing and operating future experiments.

One way to study and predict the time evolution of experiments' edge is through direct numerical simulation. This approach has been made possible with both fluid and kinetic models in open field-line turbulent systems thanks to the advent of high-performance computing (HPC). In the next few

\footnotetext{
a) Also at Princeton Plasma Physics Laboratory.; Electronic mail: manafr@mit.edu
}

years HPC will take another major step as exascale supercomputers become available, which strategies for burning plasma research plan to leverage in order to guide our understanding of current experiments and help to optimize the design of future devices ${ }^{5}$. A number of fluid and kinetic codes are currently being developed and upgraded to take advantage of these capabilities and deliver a realistic numerical description of laboratory plasmas.

Full- $f$ fluid codes, those which do not separate the evolution of equilibrium and fluctuating contributions to the plasma parameters, have modeled helical open-field line turbulence for over a decade ${ }^{6-9}$. These studies have consisted of solving a set of partial differential equations obtained from the drift-reduction of the Braginskii or Mikhailovskii fluid equations. A number of assumptions employed then have been done away with in modern Braginskii solvers, such a relaxing the Boussinesq approximation, using realistic and spatiallyvarying transport coefficients, including electromagnetic fluctuations and more complex geometries. Among the notable things uncovered by these studies are the appearance of large sheared velocity flows that quench turbulence when sources or field-line connection lengths are increased ${ }^{6}$, reminiscent of the $\mathrm{L}-\mathrm{H}$ transition in tokamaks. An early $2 \mathrm{D}$ solution of a simplified Braginskii model was also compared against experimental data, showing good agreement in several channels such as the global density and electron temperature profiles, and the frequency spectra ${ }^{7}$. In global (not field-aligned) 3D simulations, it was also possible to discern the transition from interchange to drift-wave turbulence as one lowered the pitch angle (increased connection length) and lowered the collisionality ${ }^{8}$.

As the collisionality decreases (or the temperature increases) the use of the Braginskii equations is often put into question, since these employ a short mean-free-path collisional closure to the system of moments of the kinetic equation. Despite this limitation, a variety of Braginskii codes 
have been developed recently to study the turbulence in the hot boundary plasma of tokamaks. A great effort is underway to improve the accuracy and robustness of codes such as TOKAM3 ${ }^{10}$, GBS $^{11}$, GRILLIX ${ }^{12}$, BOUT $+{ }^{13}$ and GDB ${ }^{14}$. Despite their use of collisional fluid equations in less collisional environments, several comparisons between their simulations and experiments have yielded satisfactory agreement ${ }^{15,16}$. The reduced computational cost of fluid simulations also offer the ability to perform more parameter scans and iterative numerical simulation, which is often necessary to uncover the underlying physics. Perhaps for this reason alone there may always be an interest in fluid modeling, even if only as a step prior to kinetic simulation.

Yet the possibility remains that collisionless and other kinetic effects play crucial roles in the dynamics of boundary plasmas, and that these processes cannot be captured by Braginskii fluid codes. To address such concern several teams are also developing fluid models that are not derived under the assumption of strong collisionality ${ }^{17,18}$. Extensive work is also being done in developing a new generation of solvers for the 5D gyrokinetic equation, a version of the Boltzmann kinetic equation averaged over the fast gyro-motion of particles around the magnetic field. Particle-in-cell (PIC) methods have accomplished a solution of this equation in both open and closed field lines; the XGC1 code, for example, has made valuable contributions to the prediction of heat-flux loads in current and future devices ${ }^{19}$. There is interest in cross-validating XGC1 results and also improving on its description of laboratory plasmas, for which other gyrokinetic codes are being developed, including GENE $^{20}$, GYSELA ${ }^{21}$, ELMFIRE ${ }^{22}$, PICLS ${ }^{23}$ and COGENT ${ }^{24}$. Among continuum codes, Gkeyll pioneered the simulation of gyrokinetic turbulence in open field lines ${ }^{25}$. This approach was later used to study the SOL of the National Spherical Torus Experiment (NSTX) ${ }^{26}$ and was incorporated in GENE to model the Large Plasma Device (LAPD) ${ }^{20}$.

Currently no single code has all the ingredients required for a high degree of numerical realism. By comparing these different tools, we can learn which physics are exclusively kinetic and not captured by fluid frameworks, which parameter regimes can be safely studied with fluid models, and how one description can inform the improvement of the other. There is also interest in obtaining evidence of when certain numerical or analytical simplifications make no discernible difference, or when a given theoretical assumption proves too risky.

To this end, the Helimak device serves as a helpful testbed for the description of collisional, open field-line toroidal plasmas with both fluid and gyrokinetic models. Additionally, despite the simplified geometry and relatively high collisionality of the Helimak, predictive capability is still unattained and interesting open questions remain. We thus compared simulations of this system with both the GDB two-fluid code and the Gkeyll gyrokinetic code. Although more sophisticated simulations of the Helimak are currently possible with these tools, we choose to compare the first published gyrokinetic simulations of Helimak ${ }^{27}$, with a version of GDB that incorporates some simplifications commonly used by other Braginskii codes. In section II we describe the fluid and gyrokinetic models, and some of the numerics employed to solve them. We

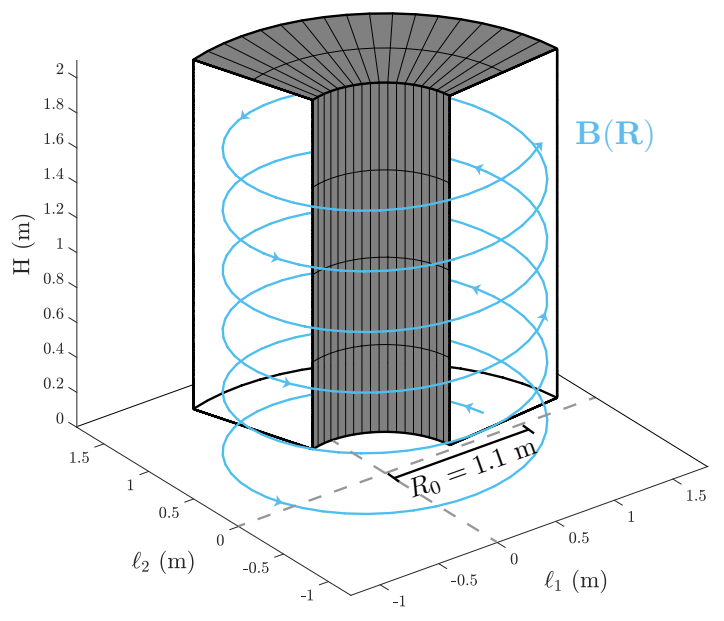

FIG. 1. Sketch of the Texas Helimak geometry. Field lines terminate on bottom and top plates, where sheath regions are located.

direct the reader to other publications for additional details on the numerical methods of $\mathrm{GDB}^{14}$ and Gkey $11^{28,29}$. The results of the fluid and gyrokinetic simulations are presented and analyzed in section III, and we offer additional discussion and conclusions in section $\mathrm{V}$.

\section{DESCRIPTION OF GYROKINETIC AND FLUID MODELS}

In this section we present the gyrokinetic and fluid models used for this study and summarize their numerical aspects. For further details on the numerical implementation we refer the reader to the documentation on $\mathrm{GDB}^{14}$ and Gkey $11^{28}$. Both codes have been used to study the Texas Helimak toroidal device consisting of a $H=2 \mathrm{~m}$ tall vessel with a rectangular cross section $1 \mathrm{~m}$ wide (see figure 1 ). The major radius in the center of the plasma is $R_{0}=1.1 \mathrm{~m}$. The background helical magnetic field $B=B(R)$, composed of a toroidal $B_{t}$ and a vertical $B_{v}$ component, starts at the bottom plate and winds counterclockwise (as seen from above) until reaching the top of the vessel. We consider experiments with large pitch angle $\left(\propto B_{v} / B_{t}\right)$ in which interchange modes with $k_{\|} \simeq 0$ dominate. Thus, as one winds around the machine once (following a field line) and displaces vertically by $L_{y}=2 \pi R B_{v} / B_{t}$, there will be little change in the plasma parameters. We can thus expect periodicity after every vertical segment $L_{y}$ long.

Gkeyll and GDB have been set up with field-aligned coordinate systems, meaning $(x, y, z)$ correspond to the radial $(R)$, binormal and field-aligned directions. The computational domain corresponds to a flux tube that begins at the bottom of the device and after $N=H / L_{y}$ turns ends at the top. The connection length is thus $L_{c}=2 \pi R N$. Both computational domains span the radial width of the vessel $(x \in[0.6 \mathrm{~m}, 1.6 \mathrm{~m}])$, the entire connection length $\left(z \in\left[-L_{c} / 2, L_{c} / 2\right]\right)$, and have a restricted periodic binormal extent $\left(y \in\left[-L_{y} / 2, L_{y} / 2\right]\right)$. Further explanation of the computational geometry can be found in a previous publication on Gkeyll simulations of this ma- 
chine $^{27}$.

We focus on an Argon case $\left(m_{i} / m_{e}=7.33 \times 10^{4}\right)$ but using the reduced mass ratio $m_{i} / m_{e}=400$, with the magnetic field magnitude $B\left(R=R_{0}\right)=B_{0}=0.1 \mathrm{~T}$ and a connection length of $L_{c}=40 \mathrm{~m}$. Given the counter-clockwise rotation of the field, the unit vector along the background magnetic field is opposite the $z$-direction: $\boldsymbol{b}=\boldsymbol{B} / B=-\hat{\boldsymbol{z}}$. We neglect the shear in the magnetic field produced by the fact that $B_{t} \propto R^{-1}$ while $B_{v}$ is constant. Other plasma parameters are close to previous experiments and numerical simulations, with the density $n_{e 0}=10^{16} \mathrm{~m}^{-3}$ and the electron temperature $T_{e 0}=10 \mathrm{eV}$. Ions do not have time to thermalize with the electrons given the rapid charge-exchange and parallel losses, so we assume low temperature ions with a source temperature of $T_{i 0}=1 \mathrm{eV}$.

\section{A. Gkeyll's gyrokinetic model}

We reproduce the description of the gyrokinetic model ${ }^{27}$ here for completeness and to motivate the choice of the sources in the fluid model. We are concerned with the electrostatic, long-wavelength limit (no Larmor-radius effects) of the full- $f$ gyrokinetic equation for the gyrocenter distribution function $f_{s}\left(\boldsymbol{x}, v_{\|}, \mu, t\right)$. This entails the continuum kinetic equation for species $s$

$$
\frac{\partial \mathcal{J}_{s} f_{s}}{\partial t}+\nabla \cdot \mathcal{J}_{s} \dot{\boldsymbol{x}} f_{s}+\frac{\partial}{\partial v_{\|}} \mathcal{J}_{s} \dot{v}_{\|} f_{s}=\mathcal{J}_{s} C\left[f_{s}\right]+\mathcal{J}_{s} S_{s}
$$

where $C\left[f_{s}\right]$ incorporates the effects of collisions via the Dougherty operator

$$
\begin{array}{r}
C\left[f_{s}\right]=\sum_{r} v_{s r}\left\{\frac{\partial}{\partial v_{\|}}\left[\left(v_{\|}-u_{\| s r}\right) f_{s}+v_{t s r}^{2} \frac{\partial f_{s}}{\partial v_{\|}}\right]\right. \\
\left.+\frac{\partial}{\partial \mu}\left[2 \mu f_{s}+2 \frac{m_{s} v_{t s r}^{2}}{B} \mu \frac{\partial f_{s}}{\partial \mu}\right]\right\}
\end{array}
$$

and $S_{S}$ is a source of particles and energy. For like-particle collisions $u_{\| s r}=u_{\| s}$ and $v_{t s r}^{2}=v_{t s}^{2}=T_{s} / m_{s}$. Ion-electron collisions are neglected and electron-ion collisions use $u_{\| e i}=u_{\| i}$ and $v_{t e i}^{2}=v_{t e}^{2}+\left(u_{\| i}-u_{\| e}\right)^{2} / 3$. The Jacobian of the coordinate transformation is $\mathcal{J}=B_{\|}^{\star}$ with $\boldsymbol{B}_{\|}^{\star}=\boldsymbol{B}+\left(B v_{\|} / \Omega_{s}\right) \nabla \times \boldsymbol{b}$ and $B_{\|}^{\star}=\boldsymbol{b} \cdot \boldsymbol{B}_{\|}^{\star}$, where $\Omega_{s}$ is the gyrofrequency of species $s$, and we simply set $\boldsymbol{B}_{\|}^{\star} \simeq \boldsymbol{B}$. Given the Poisson bracket for this Hamiltonian system

$$
\{F, G\}=\frac{B^{\star}}{m_{s} B_{\|}^{\star}} \cdot\left(\nabla F \frac{\partial G}{\partial v_{\|}}-\frac{\partial F}{\partial v_{\|}} \nabla G\right)-\frac{1}{q_{s} B_{\|}^{\star}} b \cdot \nabla F \times \nabla G,
$$

the advection velocities in phase-space are $\dot{\boldsymbol{x}}=\{\boldsymbol{x}, H\}$ and $v_{\|}=\left\{v_{\|}, H\right\}$, where the gyrocenter Hamiltonian is

$$
H_{s}=\frac{1}{2} m_{s} v_{\|}^{2}+\mu B+q_{s} \phi .
$$

There are no Larmor-radius effects so we use $\phi$ in the Hamiltonian instead of the gyroaveraged potential $\langle\phi\rangle_{\alpha}$. This gyrokinetic system is closed by the long-wavelength gyrokinetic
Poisson equation to compute the electrostatic potential:

$$
-\nabla_{\perp} \cdot \frac{n_{i 0}^{g} q_{i}^{2} \rho_{s 0}^{2}}{T_{e 0}} \nabla_{\perp} \phi=q_{i} n_{i}^{g}(\boldsymbol{x}, t)-e n_{e}(\boldsymbol{x}, t),
$$

with the ion sound gyro-radius $\rho_{s 0}=c_{s e 0} / \Omega_{i}$ given in terms of the zeroth-order ion sound speed $c_{s e 0}=\sqrt{T_{e 0} / m_{i}}$ at the reference temperature $T_{e 0}$. The guiding-center density $n_{i}^{g}$ is the zeroth velocity moment of the ion guiding center distribution function, $f_{i}$. Note that the ion guiding-center density on the left side of equation 5 is taken to be the spatially constant, reference density $\left(n_{i 0}^{g}=n_{e 0}\right)$. Similarly the variation of the magnetic field is not accounted for in the Poisson equation. This is akin to the Boussinesq approximation commonly made in Braginskii solvers.

The kinetic plasma model included the simplified phasespace source given by

$$
S_{S}\left(\boldsymbol{x}, v_{\|}, \mu\right)=S_{0} \exp \left[-\frac{\left(R-R_{\mathrm{src}}\right)^{2}}{2 \sigma_{\mathrm{src}}^{2}}\right] F_{M}\left(v_{\|}, \mu, T_{\mathrm{src}}\right) .
$$

The velocity-space variation is given by the zero-flow normalized Maxwellian with temperature $T_{\mathrm{src}}, F_{M}\left(v_{\|}, \mu, T_{\mathrm{src}}\right)$. The radial location and width of the source are given by $R_{\text {src }}$ and $\sigma_{\text {src }}$, respectively. In the experiment the heating is provided by radio-frequency $(\mathrm{RF})$ systems that interact with the electrons at the electron-cyclotron and the upper-hybrid resonances, primarily. Modeling this power source is complicated by the fact that the resonance location is dependent on the time-evolving plasma parameters. A practice of locating $S_{S}$ at a mean location of this resonance is followed here $\left(R_{\mathrm{src}}=1.0 \mathrm{~m}\right)$, and the width is chosen to be small to simulate the narrow RF source $\left(\sigma_{\mathrm{src}}=0.01 \mathrm{~m}\right)$. The absorption layer is broadened by turbulence. The appropriate amplitude of the source, and also the steady-state plasma profiles, were estimated with a 1D transport model ${ }^{26}$ assuming a balance of the particle source (for a species $s$ )

$$
S_{n, s}(\boldsymbol{x})=\frac{2 \pi B}{m_{s}} \int \mathrm{d} v_{\|} \mathrm{d} \mu S_{s}\left(\boldsymbol{x}, v_{\|}, \mu\right)
$$

and the parallel loss rate, $\left.n_{(} \boldsymbol{x}, z\right) / \tau_{\|}$, with the parallel transit time defined as $\tau_{\|}=L_{c} /\left(2 c_{s}\right)$. The result is the approximate steady-state profile

$$
n(x, z)=n_{p} \exp \left[-\frac{\left(R-R_{\mathrm{src}}\right)^{2}}{2 \sigma_{\mathrm{src}}^{2}}\right] \frac{1+\sqrt{1-z^{2} /\left(L_{c} / 2\right)^{2}}}{2}
$$

The value of $n_{p}=4.48 \times 10^{17} \mathrm{~m}^{-3}$ was set such that the volume average of $n(x, z)$ is equal to $n_{e 0}$. In order to maintain this profile with the source in equation 7 one can show that the amplitude of the source in equation 6 must be $S_{0} \approx 9.77 \times 10^{19}$ $\mathrm{m}^{-3} \mathrm{~s}^{-1}$, but Gkeyll simulations were instead carried out with $S_{0} \approx 12.98 \cdot\left(4 \times 10^{19} \mathrm{~m}^{-3}\right) \sqrt{(5 / 3)\left(T_{e}+T_{i}\right) / m_{i}} / L_{c}=$ $8.6 \times 10^{19} \mathrm{~m}^{-3} \mathrm{~s}^{-1}$. The temperature of the source's Maxwellian, $T_{\text {src }}$, was also informed by this $1 \mathrm{D}$ transport calculation, which did not include parallel heat conduction. This heat transport is significant, which is why a higher value of 
$T_{\mathrm{src}}=(10 / 3) T_{e 0}$ was employed. Since the source in equation 6 has a non-drifting distribution in velocity space there is no net external addition of momentum, but there is an injection of energy given by

$$
S_{E, s}(\boldsymbol{x})=\frac{2 \pi B}{m_{s}} \int \mathrm{d} v_{\|} \mathrm{d} \mu\left(\frac{1}{2} m_{s} v_{\|}^{2}+\mu B\right) S_{S}\left(\boldsymbol{x}, v_{\|}, \mu\right)
$$

This gyrokinetic model is discretized with high-order discontinuous Galerkin (DG) schemes. Such approach can offer increased accuracy at a reduced cost compared to other numerical techniques, can be made to adapt to complex geometries, and improves data locality which is attractive for high-performance computing. Explicit third-order RungeKutta (SSP-RK3) time stepping was used. In this work the discrete, piecewise-linear $(p=1) \mathrm{DG}$ initial conditions and sources are obtained by evaluating their analytic function at the cell boundary nodes and using linear interpolation between them (more accurate quadrature methods are also available within Gkeyll). The sources at $y=z=0$, for example, are shown in figure 2. These figures are obtained by subdividing the $x$ domain into $N_{x}(p+1)$ cells and plotting the cellcenter value of the source ( $N_{x}$ is the number of cells along $\left.x\right)$.

The boundary conditions (BCs) on the distribution function $f_{s}$ are zero-flux along $x$ and periodic in $y$. The former is consistent with a homogeneous Dirichlet condition on $\phi$, which eliminates radial flows out of the domain. In the $z$ direction a model for conducting sheath BCs with normally incident magnetic field lines are used ${ }^{25,28}$ (oblique incidence may be an interesting research topic to be examined in the future ${ }^{30}$ ). The conducting sheath is produced by solving for the potential at the sheath entrance, $\phi_{s h}=\phi\left(z= \pm L_{c} / 2\right)$, with the Poisson equation 5. Electrons with velocities $v_{\|}>\sqrt{2 e \phi_{s h} / m_{e}}$ are lost through the sheath, while those with velocity lower than this but directed towards the sheath are reflected. The ions are allowed to pass through the sheath and become absorbed at whatever velocities they are accelerated to by the potential. We simply require that there are no incoming ions from the sheath, i.e. $f_{i}\left(x, y, z=-L_{c} / 2, v_{\|}, \mu\right)=0$ and $f_{i}\left(x, y, z=+L_{c} / 2, v_{\|}, \mu\right)=0$ for $v_{\|} \geq 0$ and $v_{\|} \leq 0$, respectively.

\section{B. GDB's two-fluid model}

We employed the drift-reduced two-fluid Braginskii model. These equations are obtained via a simplification of the collisionally-closed two-fluid equations assuming time variations that are slow compared to the ion gyro-frequency, $d / d t \ll \Omega_{i}$. Under this assumption the lowest order perpendicular flows are the $E \times B\left(\boldsymbol{v}_{E}=c \boldsymbol{b} \times \nabla \phi / B\right)$ and diamagnetic flows $\left(\boldsymbol{v}_{d s}=c \boldsymbol{b} \times \nabla p_{s} /(e n B)\right)$. The reduction also invokes quasineutrality, and discards the electron polarization drift and some terms smaller by a factor of $m_{e} / m_{i}$. We also do not include collisional drift terms ${ }^{31}$. The result of such procedure $^{32}$ is the following set of equations for the time rate of change of the plasma density $n$, generalized vorticity $\omega$, mean

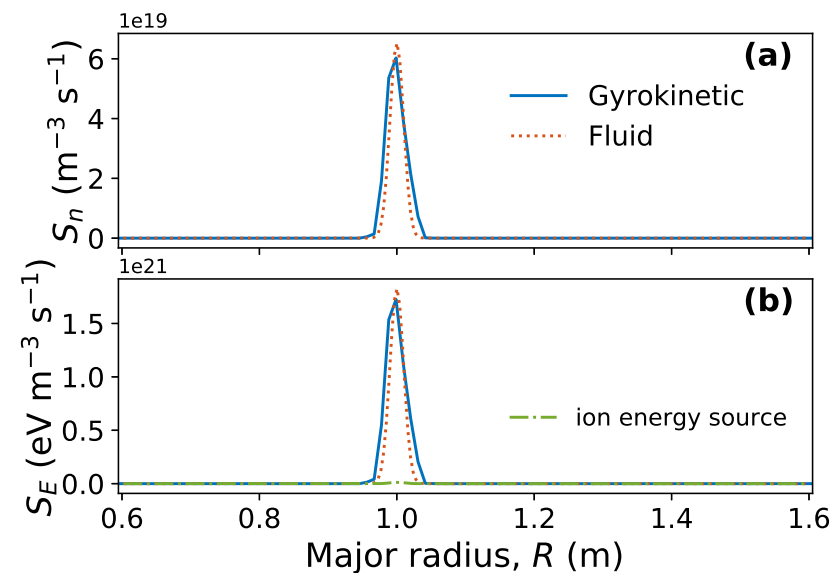

FIG. 2. Particle (a) and energy (b) sources used in the gyrokinetic and fluid simulations. Sources had no variation in $y$ and $z$.

field-aligned flows $u_{\| s}$ and temperatures $T_{s}$ :

$$
\begin{aligned}
& \frac{d^{e} n}{d t}=-\frac{2 c}{B}\left[n C_{(\phi)}-\frac{1}{e} C_{\left(p_{e}\right)}\right]-n \nabla_{\|} u_{\| e}+S_{n}+\mathcal{D}_{n} \\
& \frac{\partial \omega}{\partial t}=-\nabla \cdot\left(\frac{n c^{2}}{B^{2} \Omega_{i}}[\phi, h]+\frac{n c u_{\| i}}{B \Omega_{i}} \nabla_{\|} \boldsymbol{h}\right)+\frac{1}{e} \nabla_{\|} j_{\|} \\
& +\frac{2 c}{e B}\left[C_{\left(p_{e}\right)}+C_{\left(p_{i}\right)}\right]-\frac{1}{3 m_{i} \Omega_{i}} C_{\left(G_{i}\right)}+\mathcal{D}_{\omega} \\
& \frac{d^{e} u_{\| e}}{d t}=-\frac{1}{m_{e}}\left(\frac{\nabla_{\|} p_{e}}{n}+0.71 \nabla_{\|} T_{e}-e \nabla_{\|} \phi-e \eta_{\|} j_{\|}\right) \\
& +\frac{2 c T_{e}}{e B} C_{\left(u_{\| e}\right)}+\frac{2}{3} \frac{\nabla_{\|} G_{e}}{m_{e} n}-\frac{u_{\| e}}{n} S_{n}+\mathcal{D}_{u_{\| e}} \\
& \frac{d^{i} u_{\| i}}{d t}=-\frac{1}{m_{i}}\left(\frac{\nabla_{\|} p_{i}}{n}-0.71 \nabla_{\|} T_{e}+e \nabla_{\|} \phi+e \eta_{\|} j_{\|}\right) \\
& -\frac{2 c T_{i}}{e B} C_{\left(u_{\| i}\right)}+\frac{2}{3} \frac{\nabla_{\|} G_{i}}{m_{i} n}-\frac{u_{\| i}}{n} S_{n}+\mathcal{D}_{u_{\| i}} \\
& \frac{d^{e} T_{e}}{d t}=\frac{2}{3} \frac{T_{e}}{n}\left[\frac{d^{e} n}{d t}+\frac{1}{T_{e}} \nabla_{\|} \kappa_{\|}^{e} \nabla_{\|} T_{e}+\frac{5 n}{m_{e} \Omega_{e}} C_{\left(T_{e}\right)}+\eta_{\|} \frac{j_{\|}^{2}}{T_{e}}\right. \\
& \left.+\frac{0.71}{e}\left(\nabla_{\| j_{\|}}-\frac{j_{\|}}{T_{e}} \nabla_{\|} T_{e}\right)+\frac{1}{T_{e}} S_{E, e}-\frac{3}{2} S_{n}\right]+\mathcal{D}_{T_{e}} \\
& \frac{d^{i} T_{i}}{d t}=\frac{2}{3} \frac{T_{i}}{n}\left[\frac{d^{i} n}{d t}+\frac{1}{T_{i}} \nabla_{\|} \kappa_{\|}^{i} \nabla_{\|} T_{i}\right. \\
& \left.-\frac{5 n}{m_{i} \Omega_{i}} C_{\left(T_{i}\right)}+\frac{1}{T_{i}} S_{E, i}-\frac{3}{2} S_{n}\right]+\mathcal{D}_{T_{i}} \text {. }
\end{aligned}
$$

Note that these fluid equations appear in Gaussian units, while the gyrokinetic model is written in SI units. Previous Braginskii simulations of Helimak, and some modern 


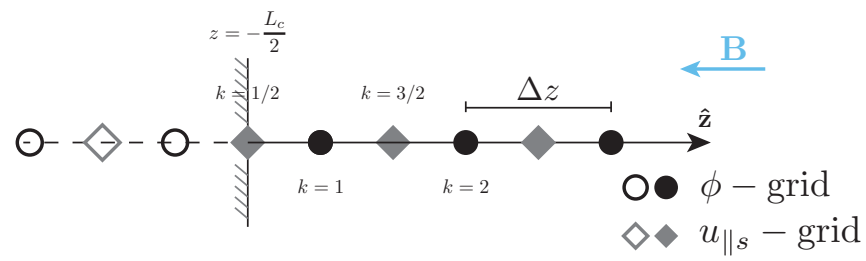

FIG. 3. Grids along the $z$ direction in GDB showing staggering of $u_{\| s}$ (diamonds) with the other quantities defined on the $\phi$-grid (circles). Here we only show a few cells near the upper sheath, at $z=-L_{c} / 2$. Cell centers are indexed by $k$.

tokamak fluid simulations ${ }^{11}$, do not evolve the ion temperature in cases where this is thought to be small but in this work we retain $T_{i}(\boldsymbol{x}, t)$. Equation 11 evolves the generalized vorticity $\omega=\nabla \cdot n c h /\left(\Omega_{i} B\right)$, written in terms of the vector $\boldsymbol{h}=\nabla \boldsymbol{\phi}+\left(\nabla p_{i}\right) /(e n)$. This equation has a contribution in terms of $G_{s}=\eta_{0}^{s}\left\{2 \nabla_{\|} u_{\| s}+c\left[C_{(\phi)}+C_{\left(p_{s}\right)} /\left(q_{s} n\right)\right] / B\right\}$, the gyroviscous part of the stress tensor with $\eta_{0}^{s}$ being the viscosity of species $s^{33}$. The time rate of change $d^{s} F / d t=$ $\partial F / \partial t+c[\phi, F] / B+u_{\| s} \nabla_{\|} F$ is given in terms of the Poisson bracket $[F, G]=\boldsymbol{b} \times \nabla F \cdot \nabla G$ and the parallel derivative $\nabla_{\|} F=\boldsymbol{b} \cdot \nabla F$. The effect of curvature of the magnetic field, $\kappa=-\hat{R} / R$, is captured by the operator $C_{(F)}=-\boldsymbol{b} \times \kappa \cdot \nabla F$. The coefficients $\kappa_{\|}^{s}$ and $\eta_{\|}$are the parallel heat diffusivity and conductivity ${ }^{33}$. We use the notation $j_{\|}=e n\left(u_{\| i}-u_{\| e}\right)$ for the parallel current.

Equations 10-15 include diffusion terms $\left(\mathcal{D}_{F}\right)$ added for numerical stability consisting of both sixth-order perpendicular and second-order parallel diffusion. The latter is not always necessary for stability, but is needed in order to produce a physical $k_{\|}$spectrum. There are also particle $\left(S_{n}\right)$ and energy $\left(S_{E, S}\right)$ sources (no momentum sources) given by

$$
\begin{aligned}
S_{n}(x, z) & =S_{0}^{\mathrm{fl}} \exp \left[-\frac{\left(R-R_{\mathrm{src}}\right)^{2}}{2 \sigma_{\mathrm{src}}^{2}}\right], \\
S_{E, e}(x, z) & =1.87 \cdot \frac{3}{2} T_{e 0} S_{n}(x, z), \\
S_{E, i}(x, z) & =0.131 \cdot \frac{3}{2} T_{i 0} S_{n}(x, z),
\end{aligned}
$$

where $S_{0}^{\mathrm{fl}}=6.525 \times 10^{19}$. The form of these fluid source was chosen to follow the plotted Gkeyll sources in figure 2. Notice that their amplitudes are lower than those obtained from equations 7 and 9 with $S_{0}=8.6 \times 10^{19} \mathrm{~m}^{-3} \mathrm{~s}^{-1}$; this is explained in section III.

The fluid equations are solved by the finite difference code GDB, and the numerical details are described in previous publications ${ }^{14,34}$. Here we only report on details of the numerical implementation relevant to the comparison with Gkeyll and pertaining the Helimak geometry. The BCs in the radial direction are homogeneous Dirichlet for $\phi$ and $\omega$, and even symmetry BCs for $n, T_{s}$ and $u_{\| s}$. These symmetric BCs are implemented by filling ghost cells so that there is symmetry about the wall surface (at $x= \pm L_{x} / 2$ ), but they do not enforce a zero-gradient at the first and last radial grid points since these are located half a grid spacing away from the wall. Hence, fluxes to the walls are allowed in GDB. The fluid code uses a grid staggered in $z$ for the parallel velocities $u_{\| s}$ (see figure 3). Therefore the cell center coordinates of the $u_{\| s}$-grid are given by $z_{k}^{u_{\| s}}=(k-1) \Delta z-L_{c} / 2$ for $k \in\left[1, N_{z}+1\right]$, while the other quantities are defined on the $\phi$-grid with coordinates $z_{k}^{\phi}=(k-1 / 2) \Delta z-L_{c} / 2$ with $k \in\left[1, N_{z}\right]$. Since we impose the lower limit of the Bohm criterion as a sheath BC for $u_{\| s}{ }^{2}$, in practice this means that at the upper sheath, for example, we set $($ recall $\boldsymbol{b}=-\hat{\boldsymbol{z}})$

$$
\begin{aligned}
& u_{\| i, k=1 / 2}=-c_{s, k=1 / 2}=-\sqrt{\frac{T_{e, k=1 / 2}+T_{i, k=1 / 2}}{m_{i}}}, \\
& u_{\| e, k=1 / 2}= \begin{cases}-c_{s, k=1 / 2} \exp \left(\Lambda-\frac{e \phi}{T_{e}}\right)_{k=1 / 2} & \phi>0 \\
-c_{s, k=1 / 2} \exp (\Lambda) & \phi \leq 0\end{cases}
\end{aligned}
$$

where $\Lambda=\log \sqrt{m_{i} /\left[2 \pi m_{e}(1+\tau)\right]}, \tau=T_{i 0} / T_{e 0}$ is the temperature ratio, and the temperatures and potential at $k=1 / 2$ are obtained via two-point linear extrapolation. We could instead impose the correct Bohm-sheath criterion $u_{\| i} \leq-c_{s}$ at this sheath by using homogeneous Neumann BCs whenever the local flow is supersonic, but the intention here is to employ techniques used by Braginskii solvers in the past. There are also more sophisticated sheath BCs accounting for oblique incidence of the magnetic field ${ }^{35}$, which will be interesting to consider in future fluid-gyrokinetic comparisons.

Evolving the values of $n, \phi$ and $T_{s}$ in the first and last cells along $z$ also requires parallel $\mathrm{BCs}$ for these quantities (and $\omega)$. While many choices exist, researchers often choose those which exhibit best numerical stability. Given that we know the direction of the flow at the sheath entrance, we fill the $z$-ghost cells (empty circles and diamonds in figure 3 ) such that an upwind stencil ensues. For example $T_{i, k=0}=3\left(T_{i, 1}-T_{i, 2}\right)+T_{i, 3}$. The calculation of parabolic terms $\left(\propto \nabla_{\|}^{2}\right)$ are computed using homogeneous Neumann BCs, except for the heat diffusivity terms discussed below.

We will distinguish between three different boundary conditions for the heat diffusivity terms $\left(\propto \kappa_{\|}^{S}\right)$ in the temperature equations. Our first choice will be to use homogeneous Neumann BCs, which lead to a zero-heat flux condition $\left(q_{\| s}=0\right)$ at the sheath. This is a common choice as it provides superior numerical stability. However, finite conductive heat-fluxes entering the sheath are measured experimentally, so we will also explore the effect of $q_{\| s} \neq 0 \mathrm{BCs}$. We implement the latter by imposing

$$
q_{\| s}=-\kappa_{\|}^{s} \nabla_{\|} T_{s}= \pm \gamma_{s} n u_{\| s} T_{s},
$$

where the upper (lower) sign corresponds to the top (bottom) sheath, and $\gamma_{s}$ is the sheath transmission coefficient. The heat transmission coefficients employed here will be $\gamma_{e}=$ $2+|e \phi| / T_{e}$ and $\gamma_{i}=2.5 T_{i} / T_{e}$. Note that this expression neglects the convective and frictional parts of the heat-flux. We will thus consider a third finite heat-flux $\mathrm{BC}$ which does account for them in the electron channel $\left(q_{\| e}^{\text {tot }} \neq 0\right)$, consisting 
of

$$
\begin{aligned}
q_{\| e}^{\text {tot }}= & n_{e} u_{\| e}\left(\frac{5}{2} T_{e}+\frac{1}{2} m_{e} u_{\| e}^{2}\right)-\kappa_{\|}^{e} \nabla_{\|} T_{e} \\
& +0.71 n_{e} T_{e}\left(u_{\| e}-u_{\| i}\right)= \pm \gamma_{e} n_{e} u_{\| e} T_{e}
\end{aligned}
$$

although in GDB the $m_{e} u_{\| e}^{2} / 2$ term was neglected as small compared to $5 T_{e} / 2$. In practice the finite heat-flux BCs are applied by filling the ghost cells accordingly; for example, at the upper sheath the $\mathrm{BC}$ in equation 18 entails

$$
\left(\ln T_{s}\right)_{k=0}=\left(\ln T_{s}\right)_{k=1}-\left.\frac{\Delta z}{\kappa_{\|}^{s}} \gamma_{s} n u_{\| s}\right|_{k=1 / 2} .
$$

Note that GDB and many other Braginskii codes evolve the logarithms of the density and the temperatures. This is a widely used technique to guarantee the positivity of such quantities. However a pitfall of this approach is that it becomes more challenging to have a conservative scheme. It is common to ameliorate the effects of non-conservation, turbulent cascades and the lack of upwinding with the use of additional numerical diffusion. Some Braginskii codes write such diffusive terms in conservative form ${ }^{36}$, but that requires either an explicit treatment or solution to a nonlinear elliptic problem. In GDB we use diffusive terms of the form ${ }^{34,37}$

$$
\mathcal{D}_{F}=\chi_{x} \frac{\partial^{6} F}{\partial x^{6}}+\chi_{y} \frac{\partial^{6} F}{\partial y^{6}}+\chi_{\|} \nabla_{\|}^{2} F
$$

discretized with second-order centered finite differences. In order to treat the perpendicular diffusion implicitly we apply it on the logarithm of $n$ and $T_{s}$.

The code solves a normalized form of equations 10-15, given in appendix A. We make several additional approximations in order to make a comparison with a fluid model representative of those used by other Braginskii solvers ${ }^{14}$. The first of these is the Boussinesq approximation, which GDB is usually run without: $\omega=\nabla \cdot n_{e 0} c h /\left(\Omega_{i 0} B_{0}\right)$. As noted in this definition of the vorticity, we will examine GDB simulations without the variation in the magnetic field amplitude, $B=B_{0}$, and will likewise use $R=R_{0}$. Often this approximation is made in the simulation of tokamak annuli because the radial extent is small, and the impact of the radial variation in $B$ is thought to be small. We also disregard the spatial variation of the $\eta_{\|}$, $\eta_{0}^{s}$ and $\kappa_{\|}^{s}$, though Gkeyll retained the spatial dependence in the collisionality: $v_{s r}=v_{s r}(x)$. These changes can modify the simulation significantly depending on which diagnostic one looks $\mathrm{at}^{32}$, but the intention here is to use assumptions and simplifications typical in Braginskii simulations found in the literature. Reporting on the effects of additional levels of complexity is left for future publication.

\section{SIMULATION RESULTS}

In this section we describe the data from the fluid and gyrokinetic simulations of the Texas Helimak, and in the following section offer additional analysis and discussion.
Gkeyll simulations used shifted-Maxwellian initial conditions (ICs) that resemble the expected steady state profiles, except for the radial density profile which followed the Gaussian source ${ }^{26,27}$. GDB was provided with similar ICs. The initial density of Gkeyll was matched in GDB with the following IC:

$$
\begin{aligned}
n(x, z, t=0)= & \frac{n_{p}}{4.9778}\left\{1.0661 \exp \left[-\frac{\left(R-R_{\mathrm{src}}\right)^{2}}{2 \sigma_{\mathrm{src}}^{2}}\right]+0.1\right\} \\
& \times \frac{1+\sqrt{1-z^{2} /\left(L_{c} / 2\right)^{2}}}{2} .
\end{aligned}
$$

A small density floor was added to avoid positivity issues in Gkeyll at early times. This initial profile was perturbed randomly with small amplitude fluctuations. The radial and fieldaligned variation of this density profile is shown in figure 4 . Also shown there is the initial parallel ion velocity, given approximately as

$$
u_{\| i}(z, t=0)=1.275 c_{s e 0} \frac{1-\sqrt{1-z^{2} /\left(L_{c} / 2\right)^{2}}}{z /\left(L_{c} / 2\right)} .
$$

The electron parallel velocity was essentially zero at $t=0$, and there was a small temperature gradient which in GDB we modeled as

$$
\begin{aligned}
& T_{e}(x, t=0)=\frac{0.96}{1+0.22 x / R_{0}} T_{e 0}, \\
& T_{i}(x, t=0)=\frac{1.17}{1+0.07 x / R_{0}} T_{i 0} .
\end{aligned}
$$

In GDB the initial vorticity was set to zero, while Gkeyll computes the initial electrostatic potential from solving the Poisson equation 5 .

These initial conditions were discretized, and the ensuing solutions obtained, on a Gkeyll phase-space mesh of $48 \times 24 \times 16 \times 10 \times 5$ cells and a piecewise-linear basis $(p=1)$, while the GDB configuration-space grid employed $256 \times 128 \times 64$ points. The velocity space domain was given by $\left[-v_{s, \max }, v_{s, \max }\right] \times\left[0,3 m_{s} v_{s, \text { max }}^{2} /\left(16 B_{0}\right)\right]$, where $v_{e, \text { max }}=$ $4 v_{t e 0}=4 \sqrt{T_{e 0} / m_{e}}$ and $v_{i, \max }=6 c_{s e 0}=6 \sqrt{T_{e 0} / m_{i}}$. The resolution of the kinetic simulation may seem coarse, but the radial spectrum of the turbulence is well converged ${ }^{27}$. The gyrokinetic simulation used 180000 CPU-hours on Skylake nodes of the Texas Advanced Computing Center's Stampede2 cluster to reach $16 \mathrm{~ms}$, while a fluid calculation required 16600 CPU-hours on MIT's Engaging cluster's Intel Xeon E5 2.1 $\mathrm{GHz}$ nodes (although the heat-flux BC choice can increase runtime by a factor of two). The cost in units of CPU-hours per milisecond, per degree of freedom was only $16 \%$ higher for Gkeyll.

\section{A. Profiles, heat-flux BCs and sourcing}

These simulations begin with a period of linear growth in which fluctuation amplitudes increase due to free energy from the pressure gradient and the curvature of the magnetic field. 


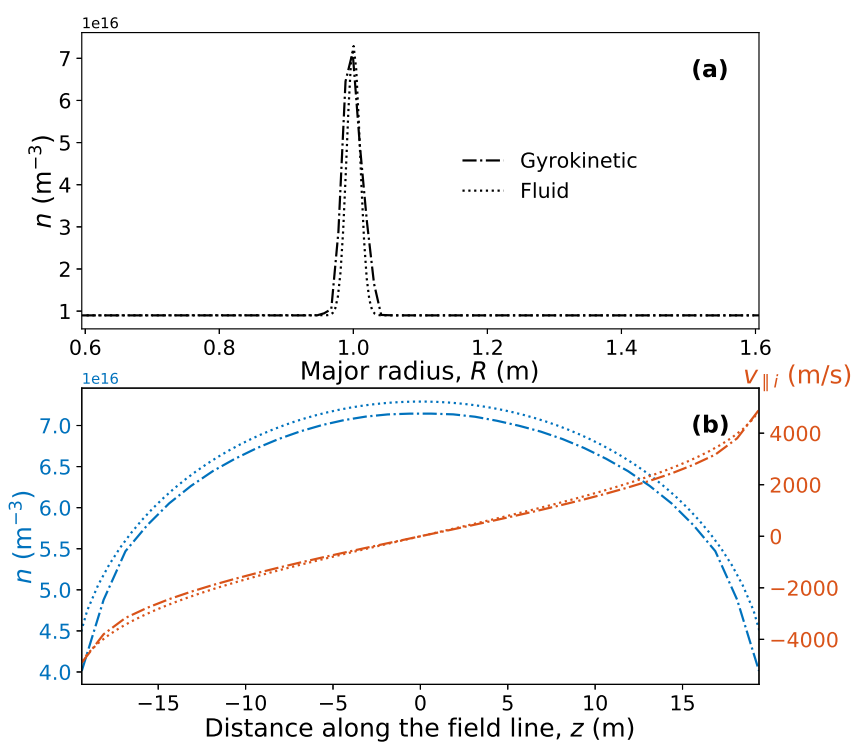

FIG. 4. Density and ion parallel velocity initial conditions in the gyrokinetic and fluid simulations. (a) Variation along $R$, and (b) fieldaligned variation.

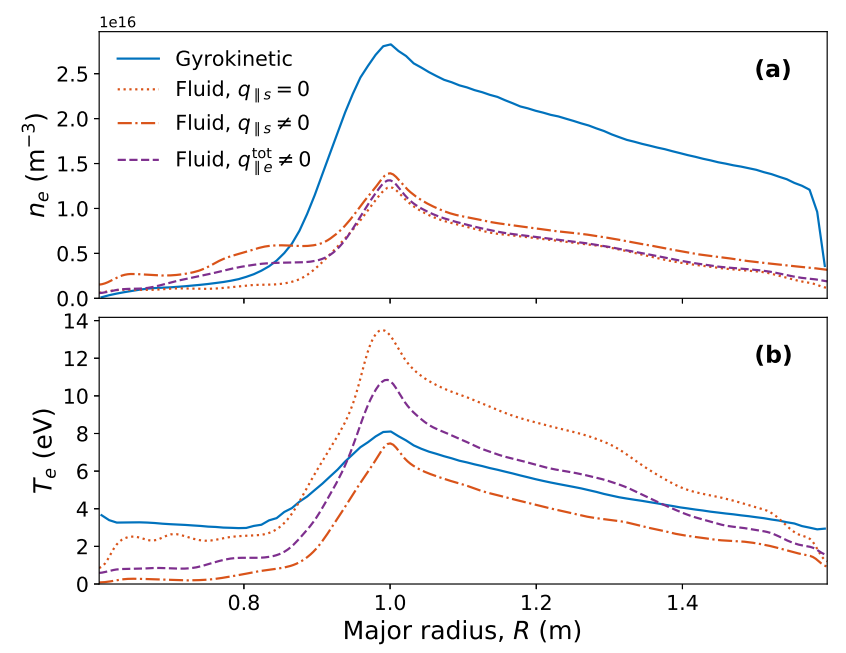

FIG. 5. Mean electron density (a) and temperature (b) profiles at $z=-L_{z} / 2$ as a function of major radius in fluid and gyrokinetic simulations. Fluid profiles are given for the simulations with boundary conditions: $\nabla_{\|} T_{s}=0$ (effectively $\left.q_{\| s}=0\right), q_{\| s} \neq 0$ and $q_{\| e}^{\text {tot }} \neq 0$.

This is typical of curvature-driven modes such as interchange or ballooning modes. Fluctuations are visually imperceptible in the short linear phase, but as they grow, they self-organize into radial streamers and mushroom or blob-like structures visible after $\sim 150 \mu \mathrm{s}$. As these formations saturate, they modify the perpendicular fluxes and alter the parallel transport. Eventually the simulation reaches a quasi-steady state in which the sources are balanced by the transport and the sheath losses and fluctuation levels saturate. Once in quasi-steady state we begin analyzing plasma profiles and turbulence properties. For example, by $y$ - and time-averaging electron density and temperature at $z=-L_{z} / 2$ we obtain the profiles in figure 5. Time averages were carried out in the $10-16 \mathrm{~ms}$ window, which is larger than the $L_{c} /\left(2 c_{s e 0}\right) \sim 4 \mathrm{~ms}$ ion transit time and at which point turbulence level is saturated. The profiles presented here, unless stated otherwise, are measured at the bottom sheath $\left(z=-L_{z} / 2\right)$ because that is one place where probe measurements are taken in the experiment.

Figure 5 shows that the peak density is roughly twice as large in the Gkeyll calculation than in the GDB simulation with $q_{\| s}=0$ BCs. There is also a drop in Gkeyll's electron density near the radial boundary which is not present in GDB. As a result of the gyrokinetic simulation approaching sheath ambipolarity the potential tends to $\phi \sim \Lambda T_{e}$ in the interior of the domain but $\phi=0$ at the boundary, so electrons near the radial boundaries are lost to the end plates very quickly because there is little sheath potential to confine them. As mentioned in section II, there are differences in the radial BCs (zero radial flux for the gyrokinetic code vs. finite radial flux for the fluid code) so there is more radial flux to offset rapid parallel losses in the fluid code than in the gyrokinetic code.

Besides differences in density profiles, figure 5 also reveals a GDB $T_{e}$ that is $67 \%$ greater than the gyrokinetic $T_{e}$. The Gkeyll sheath BCs allow a particle flux out of the simulation that carries heat with it, while our first GDB simulation explicitly imposed $\nabla_{\|} T_{s}=0$ in the heat-flux terms of temperature equations 14-15. In order to allow for a finite heat-flux into the sheath we implemented the BCs in equation 18 in GDB. This lowered the electron temperature at the sheath, and coincidentally nearly matched Gkeyll's peak $T_{e}$ (see the orange dash-dot line in figure 5b).

The considerable difference in the electron temperature of the $q_{\| s}=0$ and $q_{\| s} \neq 0$ fluid simulations is a result of the BCs in equation 18 extracting a disproportionate amount of heat. Such BCs were setting the entire electron heat flux $\left(\gamma_{e} n_{e} u_{\| e} T_{e}\right)$ to equal the conductive component, while a more appropriate $\mathrm{BC}$ takes into account the convective and frictional components as well. Such is the case of the $q_{\| e}^{\text {tot }} \neq 0$ BCs in equation 19, which effectively sets the conductive heat flux to be smaller than in the $q_{\| s} \neq 0$ simulation. The result is a slower release of heat through the sheath and thus a larger electron temperature across the plasma compared to $q_{\| s} \neq 0$ BCs (see the purple dashed line in figure 5). The gamut of zero and finite heat-flux BCs has been employed by the body of Braginskii codes in the past. Such codes sometimes set $q_{\| s}=0^{11}$, but other times they use variations of finite heat-flux BCs. For example, the $q_{\| s} \neq 0 \mathrm{BCs}$ in equations 18-20 are sometimes used in other Braginskii codes, albeit with a sheath transmission coefficient $\left(\gamma_{e}\right)$ that takes into account the $(5 / 2) T_{e}$ term in equation $19^{36,38}$. Although $q_{\| e}^{\text {tot }} \neq 0$ BCs are more accurate, in what follows we only use the $q_{\| s} \neq 0$ BCs of equation 18 because on this occasion, in combination with the other approximations made, they produced a peak fluid $T_{e}$ closer to the maximum gyrokinetic $T_{e}$.

Another observation on the consequences of $q_{\| s} \mathrm{BCs}$ is that despite the temperature drop caused by the $q_{\| s} \neq 0$ heat-sink, the peak density remained unaltered (compare orange dotted and dash-dot lines in figure 5a). Since sheath physics play an important role in this system, one might have expected that lowering $T_{e}$ would cause $c_{s}$ to decrease and, hence, the out- 


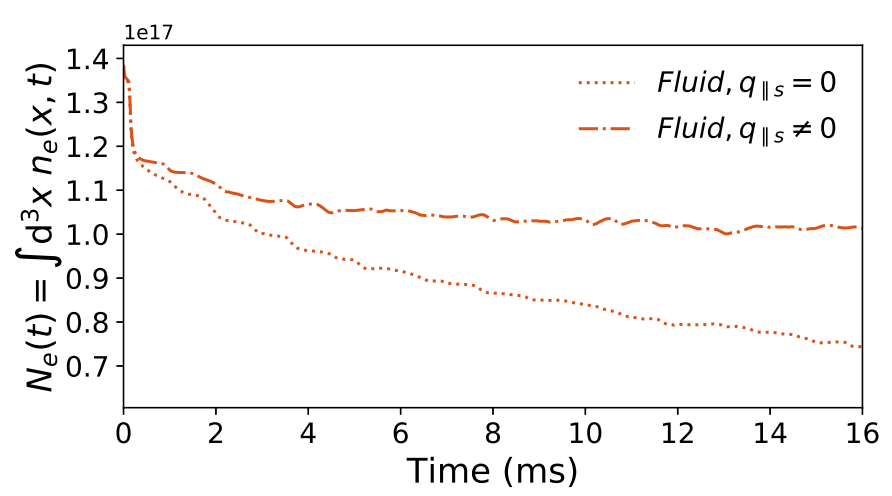

FIG. 6. Time trace of the total number of electrons in the fluid simulations with zero heat-flux (dotted line) and finite heat-flux (dash-dot line) BCs.

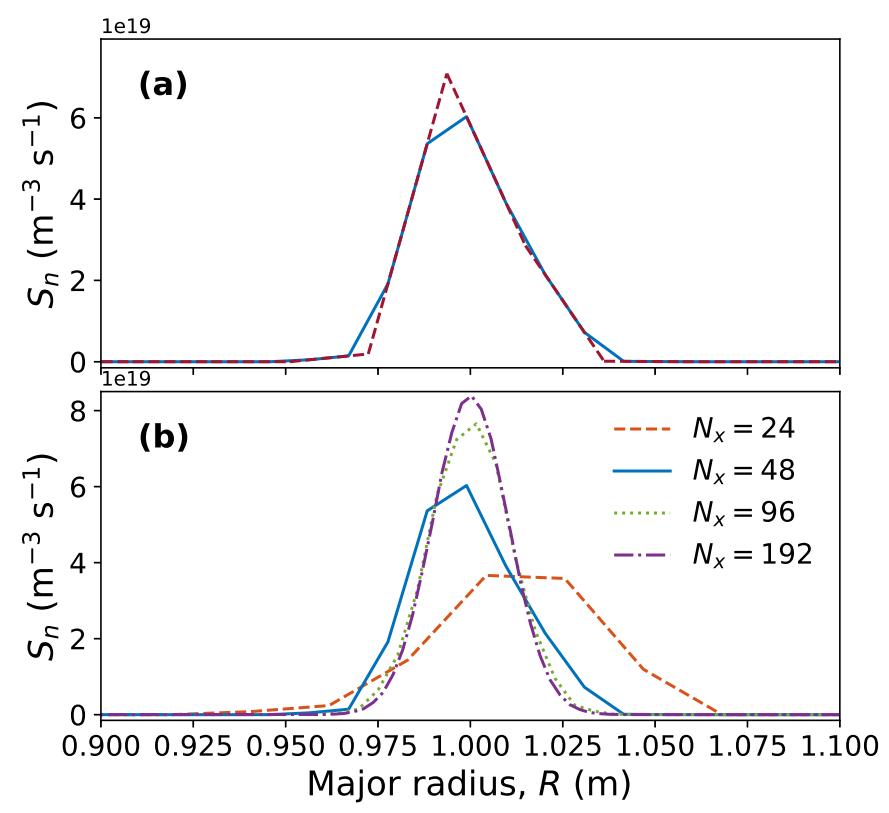

FIG. 7. (a) Gkeyll particle source $\left(S_{n}\right)$ plotted in figure 6a using cellcenter values on a grid with $N_{x}(p+1)$ (solid blue), and its discontinuous piecewise-linear representation (dashed red). (b) Gkeyll $S_{n}$ interpolated onto an $N_{x}(p+1)$ grid with varying resolution.

flow of particles to slow down and thus the density to increase. The change between the two orange lines in figure 5a does indicate an increase in the average density, and the particle loss rate did decrease when the heat-flux was allowed to be finite at the sheath entrance. This change is demonstrated in figure 6, showing the time trace of the number of electrons throughout the fluid simulations. At the end of the $16 \mathrm{~ms}$ period the $q_{\| s} \neq 0$ simulation has nearly $\sim 34 \%$ more electrons in it than the zero heat-flux counterpart. The fact that the peak density remained constant suggests that the perpendicular transport and conservation errors jointly increased to meet the weakening parallel losses.

It is still surprising that the gyrokinetic simulation yielded a much higher peak density and an entirely different den-

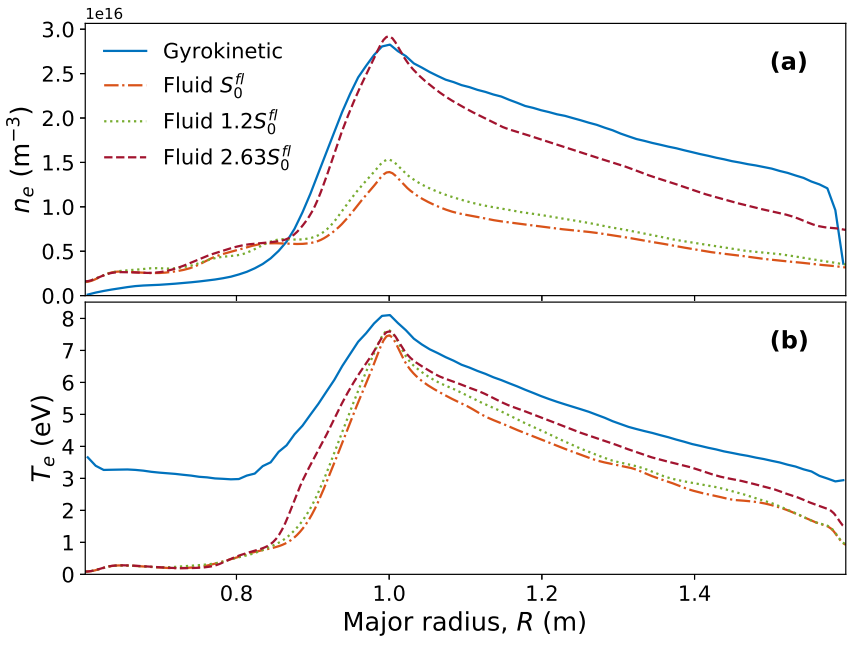

FIG. 8. Electron density (a) and temperature (b) in the gyrokinetic simulation (solid blue) compared to those in $q_{\| s} \neq 0$ fluid simulations with the sources increased by $20 \%$ and $163 \%$ from the initial $S_{0}^{\mathrm{fl}}$.

sity profile. A more careful examination of the sources reveals that the GDB simulation actually used a smaller particle fuel rate than that in Gkeyll. Part of the reason for this relates to the nature of the DG representation. The source in Gkeyll is a DG representation of equation 6 constructed by evaluating such function at cell-boundary nodes. The particle source in GDB (equation 16) was chosen to match the plotted Gkeyll source (in figure 2). The Gkeyll lines in these plots were created by evaluating the cell-center value on a grid with $N_{x}(p+1)$ cells. But note that the maximum value does not necessarily occur at these plotted coordinates, or at the cell nodes where the function was evaluated to construct the DG representation. We can plot the local piecewise linear representation (dashed red line in figure 7a) to confirm that Gkeyll actually has a higher value source than we had previously interpreted. Plotting and post-processing DG data can sometimes require subtle consideration of the underlying higher-order nature of the solution in order to avoid these errors. Another way to appreciate this nuance is by discretizing and plotting $S_{n}$ with increasing resolution (figure 7b): the amplitude converges towards the $S_{0}=8.6 \times 10^{19} \mathrm{~m}^{-3} \mathrm{~s}^{-1}$ mentioned in section II. The dashed orange line is significantly lower amplitude because at this coarse resolution the maximum of the Gaussian source lies farther from and drops off fast towards the cell-boundary nodes where equation 6 was evaluated to construct the DG representation. The plots with $N_{x}=48$ do not imply that the Gkeyll simulation used a lower source than it should have been, but rather highlight that the projection of a function onto the DG basis needs to be carefully analyzed.

Rather than roughly matching the plotted fluid and gyrokinetic sources, it is more suitable to guarantee that volume integrals of such sources agree. In order to match the volume integral of Gkeyll's density source rate (using only its cell averages and a composite trapezoidal method) it became necessary to increase the particle source rate in the fluid calcula- 
tion (equation 16) by $20 \%$, and the heat sources by $22.94 \%$. The fluid simulation was re-run with this $1.2 S_{0}^{\mathrm{fl}}$ source amplitude, keeping other parameters fixed. In figure 8 we show the effect on the time- and $y$-averaged radial density and electron temperature profiles and compare the GDB result (using $q_{\| s} \neq 0$ BCs) with Gkeyll's. Increasing the sources by $20 \%$ did not alter the electron temperature profile significantly; only slight modifications are seen across the entire radius. The peak density only increased by about $16 \%$, and still remained significantly far away from the gyrokinetic density profile. The fact that the cross-field turbulent spreading does not appear to increase, because the boundary values and profile were relatively unchanged, suggests that the parallel transport is strong and likely convects any excess particle input out the sheaths. Increasing the sources in GDB by $50 \%$ and by $125 \%$ failed to match the Gkeyll profiles. In order to approach the gyrokinetic peak density we had to augment the fluid sources by a factor of $\sim 2.63$ (163\%), shown by the red dashed line in figure 8. It is possible to slightly adjust the GDB particle and temperature sources independently in order to match the gyrokinetic peak values of both $n$ and $T_{e}$.

With the $2.63 S_{0}^{\mathrm{fl}}$ source, the difference in profile maxima for the fluid and gyrokinetic simulations is $0.6 \%$ for $n$ and $8.6 \%$ for $T_{e}$, and substantial differences can be seen in their profiles. Perpendicular particle transport more effectively widens the density profile in Gkeyll, resulting in higher densities and lower gradients on the low-field side. There is also a sharp drop in the gyrokinetic electron density at $R \approx 1.6 \mathrm{~m}$ that is absent in the GDB data, which might be caused the differences in the radial BCs; GDB is allowing radial fluxes to the wall, while Gkeyll is not (section II). On the outboard side both codes produce a similar $T_{e}$ profile, albeit shifted down by almost $1 \mathrm{eV}$ in the fluid simulation. On the high-field side, the $T_{e}$ profile is more than ten times larger in the Gkeyll data. There is a minimum of $T_{e, \min }=1.7 \mathrm{eV}$ that can be resolved by Gkeyll in order to maintain a positive distribution function with this resolution ${ }^{25}$, but even experimental data suggests $T_{e} \sim 2.5 \mathrm{eV}$ near $R \sim 0.8 \mathrm{~m}$ (see figure $8 \mathrm{~b} \mathrm{in}^{27}$ ). The extremely low GDB high-field side $T_{e}$ is caused by the choice of $q_{\| s} \neq 0$ $\mathrm{BCs}$, which as explained earlier, can cause the electrons to cool too rapidly because they neglect effects from convective and friction terms (compare dashed purple and dash-dot orange lines in figure 5). Accounting for these terms in finite heat-flux BCs, as well as using a spatially varying heat conductivity $\left(\kappa_{\|}^{e}\right)$ will substantially increase the electron temperature on the high-field side.

In addition to the differences between the gyrokinetic and the $2.63 S_{0}^{\mathrm{fl}}$ fluid simulations, we note that both fluid and gyrokinetic global profiles (figure 8) exhibit discrepancies compared to the experimentally measured, bottom-sheath equilibrium profiles ${ }^{27}$. Although the peak experimental density is close in magnitude to the simulated results, the experimental radial profiles have stronger local gradients, indicating weaker cross-field transport. Also, the experimental $T_{e}$ profile has a higher peak value located at larger radii.

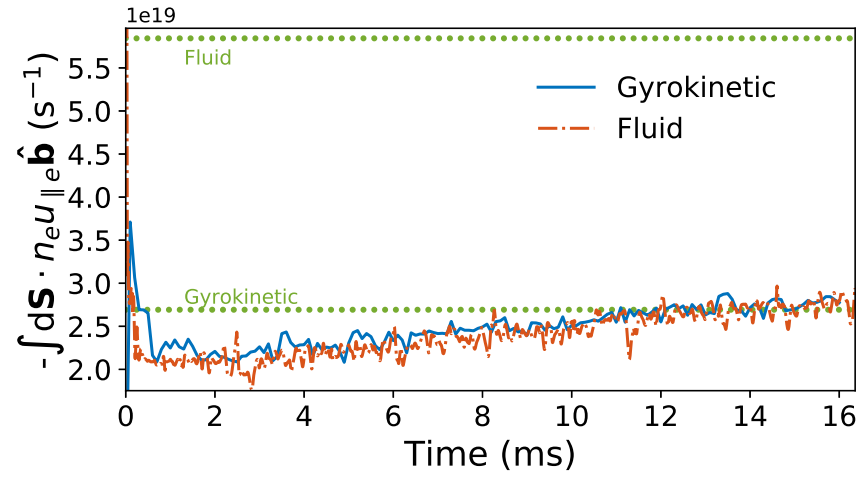

FIG. 9. Time trace of the parallel electron particle flux integrated over the sheath surface in the gyrokinetic (solid blue) and $2.63 S_{0}^{\mathrm{fl}}$ fluid (dash-dot orange) simulations. The volume integrals of their particle source is given by the green circles.

\section{B. Particle conservation in GDB}

The partial agreement we did attain in fluid and gyrokinetic density profiles came at the expense of fueling GDB 2.1875 times more strongly $\left(2.63 S_{0}^{\mathrm{fl}}\right)$, compared to the simulation that matched the volume integrated sources $\left(1.2 S_{0}^{\mathrm{fl}}\right)$. One may first suspect that the gyrokinetic model of conducting sheath BCs yields a slower outflow, but that is not the case as the integral of $n \boldsymbol{u}_{\| e}$ over the sheaths demonstrate (figure 9). In addition to the radial particle fluxes to the wall allowed in GDB, another candidate explanation for the additional particle loss is that GDB is not conservative. Formulation errors (e.g. approximations to $B(R)$ and geometric factors), discretization errors (e.g. from non-conservative finite differences using $\ln n_{e}$ instead of conservative finite differences using $n_{e}$ ), and numerical diffusion can conspire to break particle conservation. Figure 9 is evidence that non-conservation errors can be $\mathcal{O}(1)$; even though the volume integrated density is in quasi-steady state the parallel flux to the end plates is only about half of the input source in the fluid code, meaning that the other half of the particles are being lost mostly due to some other errors, either due to the formulation of the fluid equations or numerical errors.

The aforementioned diffusion terms are nonetheless thought to be small in these GDB runs (see reported values in appendix A), but its true impact depends on the resolution and the turbulent scales generated by the regime one is simulating. We can examine the volume integral of each of the terms in the density equation (figure 10) and confirm the relative smallness of the diffusion terms (blue diamonds, although in this diagnostic the contribution from perpendicular diffusion was computed using the density outputted every 400 time steps and is likely to differ some from the impact of the true, implicit perpendicular diffusion). This analysis also confirms a concerning imbalance between parallel losses and sources: the former only accounts for $54 \%$ of the latter towards the end of the simulation (compare orange dash-dot line and green circles). This 


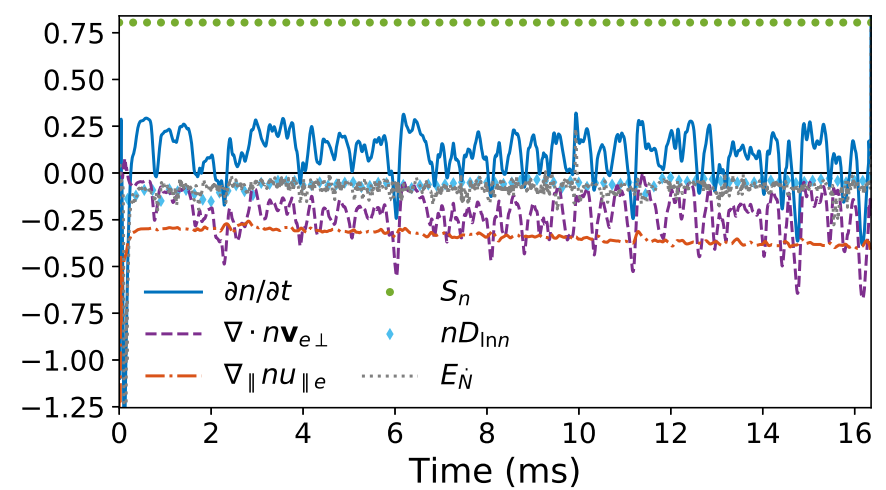

FIG. 10. Volume integrated terms in the normalized density equation 10 in the GDB simulation with $q_{\| s} \neq 0$ and $2.63 S_{0}^{\mathrm{fl}}$ (see normalization in section $\mathrm{A})$. The blue line $(\partial n / \partial t)$ is computed using a centered finite difference in time between snapshots $6.06 \mu \mathrm{s}$ apart, and the grey dotted line shows $\partial n / \partial t$ minus all the other lines. A $96 \mu \mathrm{s}$ moving average was applied to all lines before plotting.

gap is all the more puzzling since the particle accounting error

$$
\begin{aligned}
E_{\dot{N}}=\left\langle\left.\frac{\partial n}{\partial t}\right|_{F D T}\right\rangle_{x y z}-( & -\left\langle\nabla \cdot n v_{e \perp}\right\rangle_{x y z}-\left\langle\nabla_{\|} n u_{\| e}\right\rangle_{x y z} \\
& \left.+\left\langle S_{n}\right\rangle_{x y z}+\left\langle n \mathcal{D}_{\ln n}\right\rangle_{x y z}\right)
\end{aligned}
$$

with the first term measured with finite differences in time and $\nabla \cdot n \boldsymbol{v}_{e \perp}=(c / B)[\phi, n]+(2 c / B)\left[n C_{(\phi)}-C_{\left(p_{e}\right)} / e\right]$, appears to be negligible (dotted grey line). This seemingly small error is only possible because $\partial n / \partial t$ is on average not zero, and because the volume integral of $\nabla \cdot n \boldsymbol{v}_{e \perp}$ does not vanish despite the BCs on $\phi$ (homogeneous Dirichlet in $x$ and periodic in $y$ ).

A non-vanishing $\int \mathrm{d}^{3} \boldsymbol{x} \nabla \cdot n \boldsymbol{v}_{E}$ may indirectly affect the parallel sheath losses, so we are interested in ensuring this basic feature. We illustrate this with a concrete simpler example, neglecting the vertical field in the Helimak and considering a purely toroidal field with $B$ in the $-\varphi$ direction over a small, periodic extent in $y$ confined by perfectly conducting walls in $x$. Adopt a coordinate system $(x, y, z)$ related to cylindrical coordinates by $(x, y, z)=\left(R, Z,-R_{0} \varphi\right)$, where $Z$ is the vertical coordinate and $\varphi$ the toroidal angle. In this case the volume element is then $\mathrm{d} R \mathrm{~d} Z R \mathrm{~d} \varphi$ and we write this as $\mathrm{d} R \mathrm{~d} Z R_{0} \mathrm{~d} \varphi\left(R / R_{0}\right)=\mathrm{d} x \mathrm{~d} y \mathrm{~d} z\left(R / R_{0}\right)=\mathrm{d}^{3} \boldsymbol{x}\left(R / R_{0}\right)$. The $E \times B$ particle balance entails

$$
\begin{aligned}
& \frac{\partial N_{e}}{\partial t}=-\int \mathrm{d}^{3} x \frac{R}{R_{0}}\left\{\frac{c}{B}\left[\phi, n_{e}\right]+\frac{2 c}{B} n_{e} C_{(\phi)}\right\}, \\
&=-\int \mathrm{d}^{3} x \frac{R}{R_{0}} \frac{c}{B}\left\{\left(\frac{\partial \phi}{\partial y} \frac{\partial n_{e}}{\partial x}-\frac{\partial \phi}{\partial x} \frac{\partial n_{e}}{\partial y}\right)+2 n_{e} C_{(\phi)}\right\}, \\
&=-\int \mathrm{d}^{3} x \frac{R}{R_{0}} \frac{c}{B}\left\{\left[\frac{\partial}{\partial x}\left(\frac{\partial \phi}{\partial y} n_{e}\right)-\frac{\partial}{\partial y}\left(\frac{\partial \phi}{\partial x} n_{e}\right)\right]\right. \\
&\left.+\frac{2}{R} n_{e} \frac{\partial \phi}{\partial y}\right\}, \\
&=-\int \mathrm{d} x \mathrm{~d} y \mathrm{~d} z \frac{R}{R_{0}}\left[\frac{c}{B} \frac{\partial}{\partial x}\left(\frac{\partial \phi}{\partial y} n_{e}\right)+\frac{2 c}{B R} n_{e} \frac{\partial \phi}{\partial y}\right],
\end{aligned}
$$

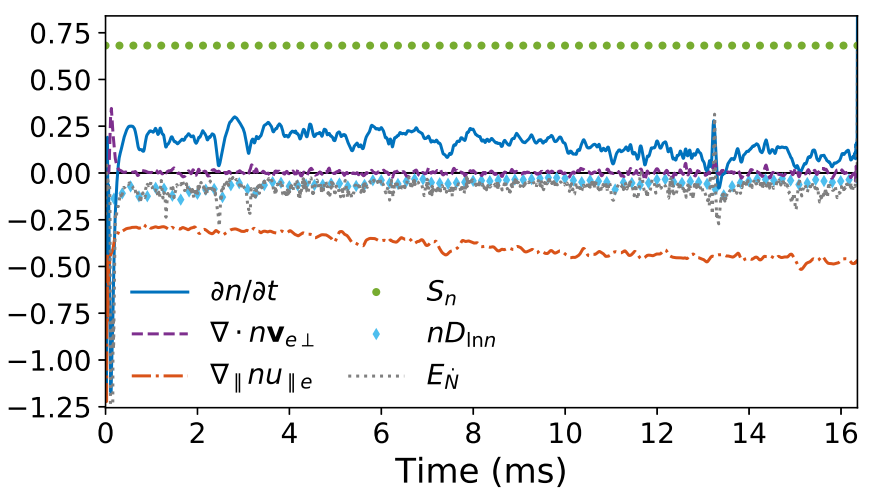

FIG. 11. Similar to figure 10, this plot shows volume integrated terms in the normalized density equation 10 , but for a GDB simulation with $q_{\| s} \neq 0,2.45 S_{0}^{\mathrm{fl}}$ and keeping $R=R(x)$ and $B=B(R)$ as the analysis in equation 26 suggests. We can see that by including these dependencies the volume-integrated perpendicular flux does vanish. A lower source amplitude $\left(2.45 S_{0}^{\mathrm{fl}}\right)$ was chosen because it was sufficient to produce a comparable GDB simulation with a plasma in a similar parameter regime as the Gkeyll simulation.

where we made use of the periodic BCs along $y$ and the equality of mixed partials. For particle number to be conserved in this isolated $E \times B$ system it must be that the first term in equation 26 cancels the second.

In the Helimak scenario such cancellation leads to the balance between sources and parallel losses, but this balancing was only partial in GDB because of modifications to the treatment and analysis of the $E \times B$ terms compared to what is in equation 26. The equations used in GDB so far account for the variation in $B$ in the curvature term $(1 /(B R)=1$ in the second term of equation 26), but they replace $B \rightarrow B_{0}$ in the $E \times B$ nonlinearities $\left(c / B \rightarrow c / B_{0}\right.$ in the first term of equation 26). This approximation was invoked in some annulus studies of tokamak SOLs because the variation in $R$ across that domain was thought to be small ${ }^{39,40}$. It is often thought to be a small correction on the turbulent time-scale $\sqrt{L_{p e} R / 2} / c_{s e 0} \simeq \sqrt{0.27 \mathrm{~m} \cdot 1.2 \mathrm{~m} / 2} /\left(3.5 \times 10^{3} \mathrm{~m} / \mathrm{s}\right)=0.115$ $\mathrm{ms}$, but these errors build up and become significant on the confinement time scale $L_{c} / c_{s} \approx 8 \mathrm{~ms}$. Furthermore, as Braginskii codes increasingly model larger radial domains the thin annulus approximation incurs ever larger inaccuracies. For the Helimak case presented here $B$ changes by a factor of $8 / 3 \approx 2.7$, and setting $1 / B=1$ in the $E \times B$ term and $R / R_{0}=1$ in the equivalent particle balance shown in figure 10 causes the volume integrals of $n \nabla \cdot \boldsymbol{v}_{E}$ and $\boldsymbol{v}_{E} \cdot \nabla n$ to not cancel each other, and $\int \mathrm{d}^{3} \boldsymbol{x} \nabla \cdot n \boldsymbol{v}_{e \perp}$ to not vanish. We can estimate the relative size of this error contribution, defining $\langle f\rangle=\int \mathrm{d}^{3} \boldsymbol{x} f /\left(\int \mathrm{d}^{3} \boldsymbol{x}\right)$, as

$$
\frac{\int \mathrm{d}^{3} \boldsymbol{x} \frac{2 c}{B R} n_{e 1} \frac{\partial \phi}{\partial y}}{\int \mathrm{d}^{3} x \nabla_{\|} n_{e} u_{\| e}} \sim \frac{L_{x} L_{y} L_{c}\left\langle\frac{2 c}{B R} n_{e 1} \frac{\partial \phi_{1}}{\partial y}\right\rangle}{2 L_{x} L_{y} n_{e 0} c_{s e 0}} \sim \frac{\rho_{s}}{R} \frac{L_{c}}{L_{\perp}}\left\langle\frac{n_{e 1}}{n_{e 0}} \frac{e \phi_{1}}{T_{e 0}}\right\rangle,
$$

which can result in $\mathcal{O}(1)$ accumulated errors (we assumed maximally out of phase density and potential fluctuations of the same magnitude and estimated $\partial_{y} \phi_{1} \sim L_{\perp}^{-1} \phi_{1}$, with $L_{\perp}$ a 
characteristic fluctuation perpendicular length scale). Note that even in a tokamak SOL, where $\rho_{s} / R$ is small, this error can be significant because the parallel connection length can be very large and $\mathcal{O}(1)$ perturbations can occur. Had we also included the $1 / B_{(R)}$ factors in the $E \times B$ nonlinearities we would find that the diffusion terms and the errors in particle balance (equivalent of blue diamonds and dashed grey line in figure 10) account for about $21 \%$ of the plasma injected, the non-zero $\partial n / \partial t$ for $\sim 9 \%$ and the rest is lost to the sheaths (see figure 11). For context note that the latest version of most Braginskii fluid codes have the capability to keep the full Jacobian factors that improve conservation properties, and in recent years there has been more attention paid to implementing fluid algorithms with good conservation properties ${ }^{10-12,41}$.

\section{Comparison of flows and turbulence in GDB and Gkeyll}

The $R \rightarrow R_{0}$ approximation made in these GDB simulations (except for that in figure 11) had been used in some previous Braginskii turbulence work ${ }^{39,40}$, and that with an enhanced source of $2.63 S_{0}^{\mathrm{fl}}$ produced approximately the same regime as the gyrokinetic simulation. Hence, in what follows we compare Gkey 11 results with such GDB simulation $\left(2.63 S_{0}^{\mathrm{fl}}, q_{\| s} \neq 0\right.$ and $1 / B \rightarrow 1$ in $E \times B$ convective terms) in more detail. A snapshot of the plasma density, electron temperature and electrostatic potential at $10 \mathrm{~ms}$ is given in figure 12 (colors set by the extrema in the gyrokinetic data). Instantaneous maxima can be very different in the two simulations even if the $y$ and time averaged profiles have similar maximum values, hence the bright yellow region in the snapshot of the fluid density. This maximal region suggests that radial transport is weaker there, while the Gkeyll simulation appears to spread out the plasma radially more effectively. This is consistent with the average $n$ profile in figure 8 . Furthermore, fluctuations in GDB seem have finer-scale structure, suggestive of a different $k_{\perp}$ spectrum and perhaps a smaller correlation length. The difference in radial turbulent spreading is also visible in the $T_{e}$ snapshot of figure 12 . The right-most column of this figure depicts relatively smooth $\phi$ profiles that do not resemble the plasma density fluctuations, indicating a significant departure from adiabaticity.

Looking at these snapshots a reader may be inclined to think that the gyrokinetic simulation is not well-resolved or that it is too diffusive. The smoother, larger perpendicular scales of the fluctuations in the gyrokinetic pictures of figure 12 are likely not a product of numerical diffusion since Gkeyll is a conservative code. Though the spatial resolution of Gkeyll is less than GDB's, the convergence of the radial $\left(k_{x}\right)$ spectrum upon grid refinement ${ }^{27}$ and the similarities with GDB in quantities compared below suggests that the resolution is sufficient. It is still possible that metrics other than the $k_{x}$ spectrum would have shown greater variance. This was not explored exhaustively, and higher resolution simulations with the new, faster version of Gkeyll may shed light on this point. Readers may recall, however, that it is longer wavelength modes that tend to drive most of the transport, and achieving fine-scale agreement between Gkeyll and GDB may only affect the more intricate details of the turbulence.

The strength of the turbulent fluctuations is however different in GDB and Gkeyll at the mid-plane $(z=0)$, at least as far as the relative root-mean-square (RMS) fluctuations in the saturation current $\left(I_{\mathrm{sat}}\right)$ is concerned. Following experimental convention, instead of the density we use $I_{\text {sat }}=n_{e} \sqrt{T_{e}}$ in computing density fluctuations, but for simplicity refer to it as $\delta n$ unless stated otherwise. Figure 13 provides a calculation of the $z=0 \delta n$ RMS fluctuation amplitude calculated using the instantaneous $y$-average, $\delta n=n-\langle n\rangle_{y}$, and normalized to $\langle n\rangle_{x y}$. We see that at $z=0$ and computing $\delta n$ using the instantaneous $y$-average yields a $\delta n_{\mathrm{rms}} /\langle n\rangle_{x y}$ that is twice as high in the fluid than in the gyrokinetic simulation. We will later see that this may be dominated by fluctuations levels in the high-field side of the fluid simulation, on which parallel heatflux BCs have a significant impact. These are also considerably higher fluctuation levels than in previous simulations, using simpler fluid models, of Helimak ${ }^{7}$.

Such qualitative differences arise throughout movies of the plasma density, yet these videos also reveal similarities in the binormal flows of both simulations. We obtained the time and $y$-averaged binormal component of the $E \times B$ drift velocity from both simulations and plot them in figure 14. These sub-sonic flows are particularly comparable on the low-field side where most of turbulence is located. The maximum $v_{E}$ is only $6 \%$ higher in the fluid simulation and is located $3 \mathrm{~cm}$ farther out than the $R=1.2 \mathrm{~m}$ location of the gyrokinetic peak $v_{E}$. An estimate of the experimental $E \times B$ profile using $\phi_{\exp }=\Lambda T_{e, \exp } / e$ leads to the conclusion that both the fluid and gyrokinetic simulation produce a $v_{E}$ that is quite different from that in the experiment ${ }^{27}$. As explained in such previous work, there is an important vertical component to the $E \times B$ flow that can be larger than the vertical projection of the parallel sonic flows. Incorporating these effects would require a more accurate description of the geometry and is beyond the scope of this work.

Continuing with the examination of flows, we compared parallel ion velocities from the GDB and Gkeyll simulations by averaging $u_{\| i}$ in $y$ and time at $R=1.24 \mathrm{~m}$ (figure 15). Near the center of the $z$-domain, $u_{\| i}$ is nearly equivalent in both models, but there are slight differences in gradients and more significant differences near the sheath. The larger $u_{\| i}$ gradient in the fluid simulation may seem suggestive a stronger particle outflow and thus a need for greater source rates, but as we now know the integrated particle flux out is similar for both codes (figure 9). Ultimately the parallel losses are set by the exit value of the flux, which depends on a non-trivial density profile and the exit parallel velocity. The latter is forced to $u_{\| i}= \pm c_{s}$ in GDB, while Gkeyll's conducting sheath model does not enforce the Bohm criterion, which could explain the differences in $u_{\| i}$ near the sheath. In this case, the gyrokinetic parallel ion flow at the sheath entrance was $23 \%$ lower than the local $y$ and time averaged value of the sound speed $\left(T_{i} \ll T_{e}\right.$ there).

A more complete picture is developed by considering the variation of $u_{\| i}$ across the radius of the machine. Figure 16 illustrates that departures from the Bohm criterion in the gyrokinetic simulation are even greater near the source region 

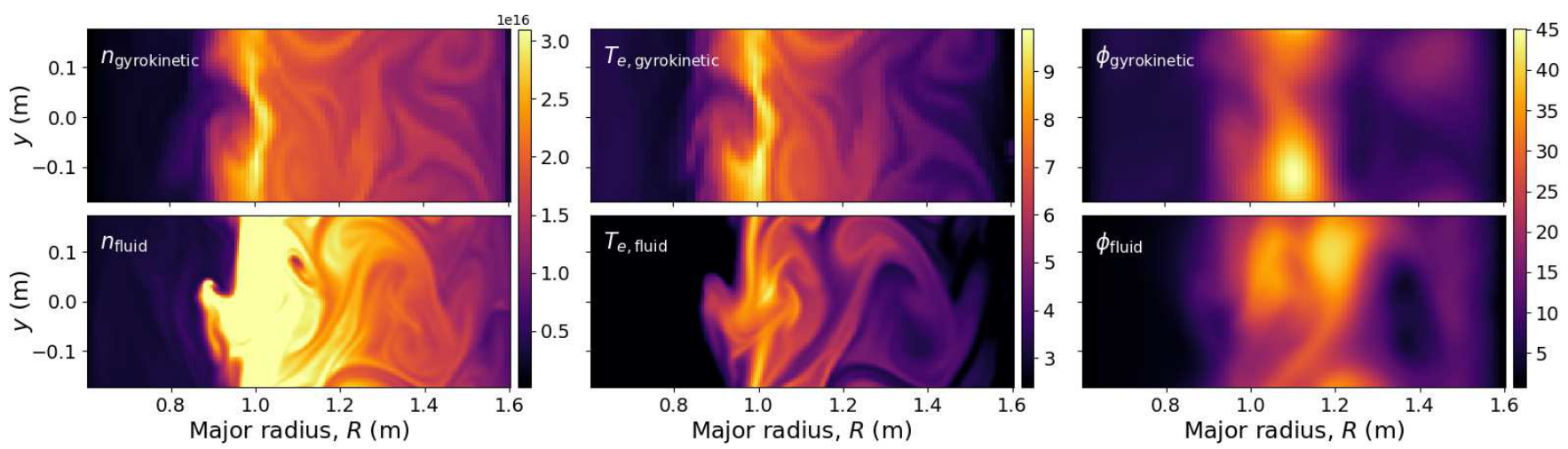

FIG. 12. Snapshots of the $z=0$ plasma density (left column, in $\mathrm{m}^{-3}$ ), electron temperature (center column, in eV) and electrostatic potential (right column, in volts) in the gyrokinetic (top row) and fluid (bottom row) simulations at $t=10$ ms. Colors scaled by gyrokinetic data.

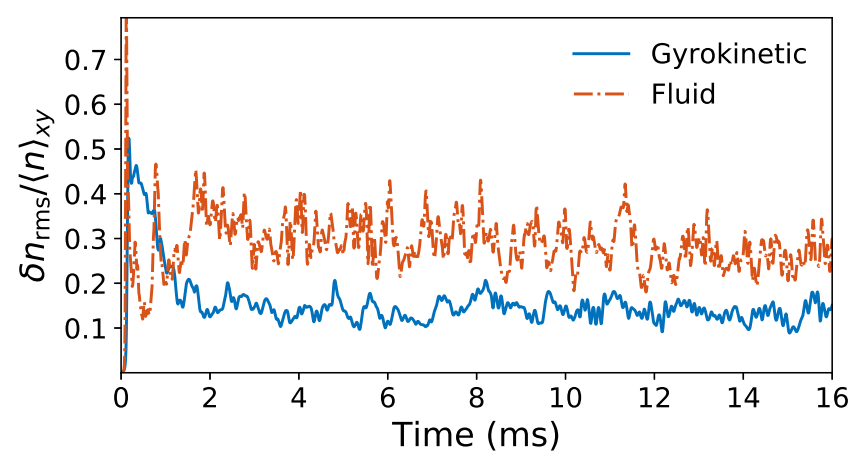

FIG. 13. Relative root-mean-square of the density fluctuations in the $z=0$ plane as a function of time. The saturation current $I_{\text {sat }}=n \sqrt{T_{e}}$ is used as a density proxy as is typically done with experimental probe data. This fluid simulation used $q_{\| s} \neq 0$ and $2.63 S_{0}^{\mathrm{fl}}$.

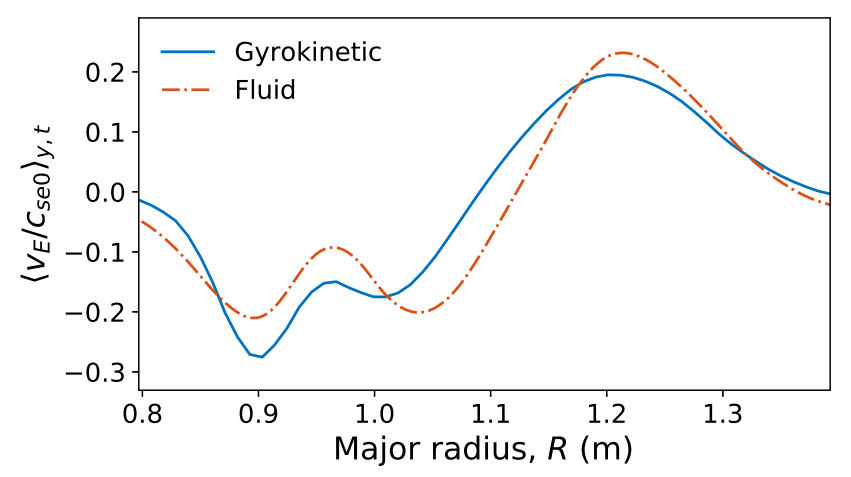

FIG. 14. Binormal component of the $E \times B$ drift normalized to the reference sound speed, averaged over $y$ and time.

(compare solid blue line with blue crosses). The difference between the fluid $u_{\| i}$ and $c_{s e}$ at $R<0.9 \mathrm{~m}$ is a consequence of enforcing $u_{\| i}= \pm c_{s}=\sqrt{\left(T_{e}+T_{i}\right) / m}$ and that $T_{i}>T_{e}$ in the high-field region. On the other side, in the low-field region where most of the plasma is found, GDB's $u_{\| i}$ is almost consistently greater than that in Gkeyll. Therefore, the par-

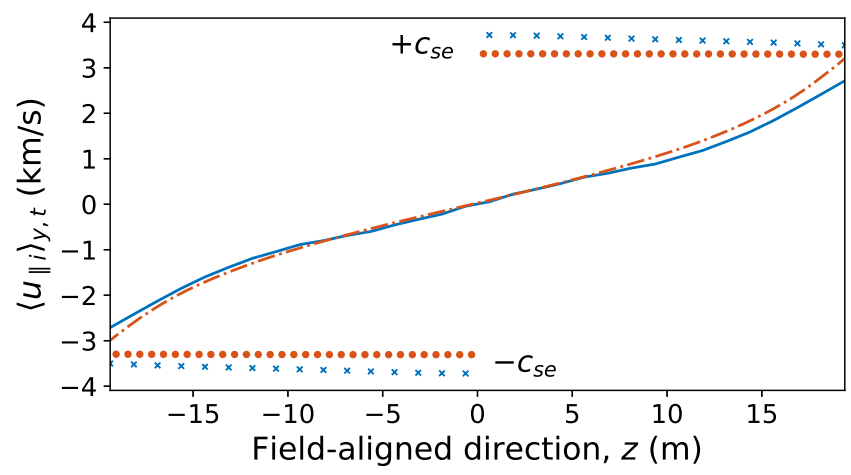

FIG. 15. $y$ and time averaged ion parallel velocity at $R=1.24 \mathrm{~m}$ in the gyrokinetic (solid blue) and fluid (orange dash-dot) simulations. We also show the time and $y$ averaged zeroth-order sound speed (at the same $R$ ) in the gyrokinetic (blue crosses) and fluid (orange circles) calculations.

allel transit $L_{c} /\left(2 u_{\| i}\right)$ is slower in the gyrokinetic simulation and the plasma has more time to transport radially across field lines, in agreement with the average density profiles and turbulent snapshots presented above.

Weaker parallel flows are generally associated with more cross-field transport. However, the gyrokinetic simulation shows lower turbulence levels than the fluid simulation as measured by the relative RMS fluctuations using the instantaneous $y$-average at $z=0$ (figure 13). The presence of finescale structures in the $y$ direction of the fluid simulation seen in figure 12 does not explain this discrepancy since lower $k_{y}$ modes tend to drive most of the transport. We, therefore, compared the $\delta n_{r m s}$ radial profile in figure 17 using $\delta n=n-\langle n\rangle_{y, t}$, i.e. the time and $y$ average instead of the instantaneous $y$ average alone. The peak relative fluctuation level occurs at the same location for both the fluid and gyrokinetic simulations, though it is $25 \%$ higher in GDB and it occurs on the high-field side where many other discrepancies between the codes are seen (e.g., time-averaged profiles in figure 8). On the low-field side Gkeyll's relative $\delta n_{r m s}$ is in fact slightly lower than GDB's, so the more effective cross-field spreading of the gyrokinetic density is likely a more direct consequence 


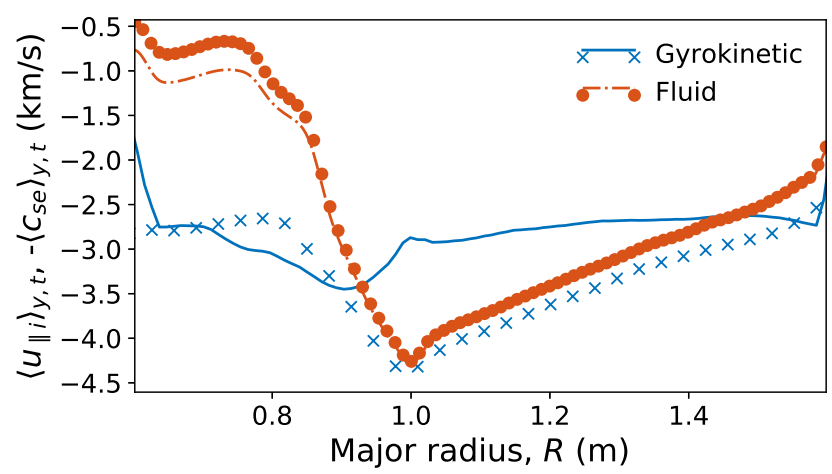

FIG. 16. Ion parallel velocity, $y$ and time averaged, at the bottom sheath as a function of radius in the gyrokinetic (solid blue) and fluid (orange dash-dot) simulations. Also shown is the time and $y$ averaged zeroth-order sound speed in the gyrokinetic (blue crosses) and fluid (orange circles) calculations.

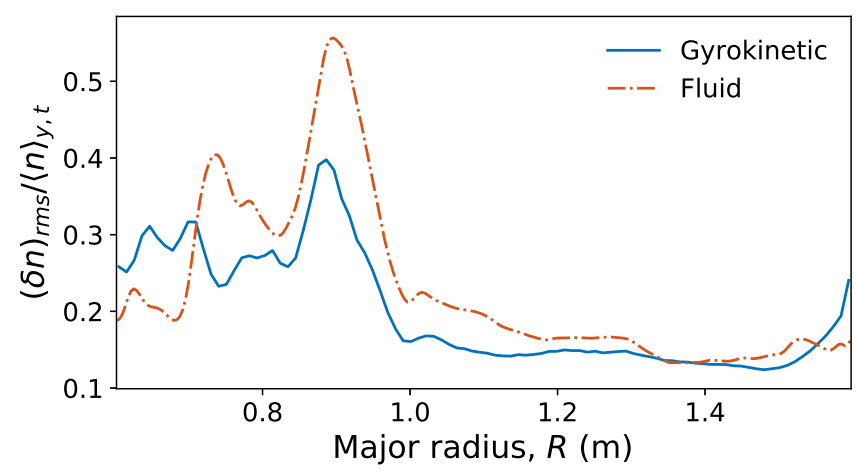

FIG. 17. Relative root-mean-square fluctuation density profile for gyrokinetic (solid blue) and fluid (orange dash-dot) simulations.

of the difference in the flows.

These RMS fluctuation profiles are nevertheless qualitatively similar: peaking in the high-field region and relatively flat in the outboard side. Fluctuations also have comparable qualities in the parallel direction. Take the power in the $k_{z}$ spectra of the density fluctuations in both simulations, for example (figure 18, using density fluctuations computed with $\left.\delta n=n-\langle n\rangle_{y}\right)$. The Gkeyll spectrum was computed by Fourier transforming cell-average values. The $y$ - and timeaveraged spectra in figure 18 both decay rapidly beyond $k_{z}=$ 0 . This $k_{z} \approx 0$ feature is characteristic of the interchange turbulent regime, which is predicted for the high field-line pitch angle used in these simulations ${ }^{8}$. The lack of power in high $k_{z}$ modes is also observed in snapshots of the plasma density in $x-z$ at $t=10$ ms (figure 19), with both simulations showing little variation along field lines.

The fluid $k_{z}$ spectrum was higher than Gkeyll's for all finite $k_{z}$ modes, though we note that GDB's spectra can be altered artificially by the use of additional numerical parallel diffusion. The GDB code is able to run with $\chi_{\|}=0$ but a centered finite-difference scheme without any upwinding can generate more $k_{z} \neq 0$ structures, significantly altering the parallel spectrum. Hence, small parallel diffusion terms were included, as

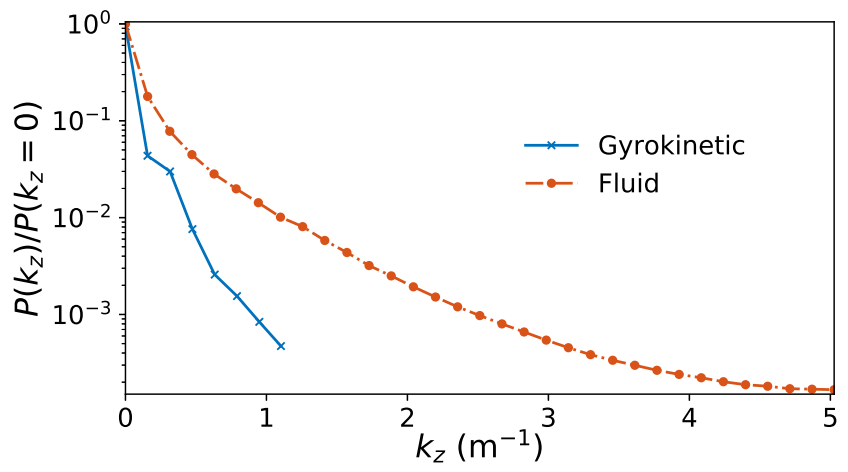

FIG. 18. Spectra of the density fluctuations along $z$, averaged over $y$ and time. The gyrokinetic spectra was also averaged over $x$, while the fluid data only averaged the spectra at the locations $R=0.83 \mathrm{~m}$, $R=1.0 \mathrm{~m}$, and $R=1.24 \mathrm{~m}$.

indicated in appendix A. Finite parallel diffusion has stability benefits and is also used to regularize the $k_{z}$ spectrum, though it was not adjusted deliberately to match Gkeyll results.

We also observed similarities in frequency-domain power distribution. As done in previous analysis of Gkeyll data ${ }^{27}$, we multiplied the time signal of the fluctuations $\delta n=n-\langle n\rangle_{t}$ by a Hann window

$$
\delta n_{w}\left(t_{i}\right)=\left[1-\cos \left(\frac{2 \pi}{N_{t}-1} i\right)\right] \delta n\left(t_{i}\right)
$$

to account for the fact that the fluctuation data is not periodic at the first and last time frames, $t_{0}$ and $t_{N_{t}-1}$, respectively. Upon Fourier transforming this quantity to the frequency domain, we compute the normalized power spectra via

$$
P(f)=\frac{\left\langle\left|\delta n_{w}(f)\right|^{2}\right\rangle}{\sum_{f}(\Delta f)\left\langle\left|\delta n_{w}(f)\right|^{2}\right\rangle} .
$$

By using a $6 \mathrm{~ms}$ time signal, we resolved the frequency domain with a frequency spacing of $\Delta f=168 \mathrm{~Hz}$ in Gkeyll and $\Delta f=166 \mathrm{~Hz}$ in GDB. The frequency spectra (multiplied by the frequency) given in figure 20 were computed at the location of maximum $E \times B$ drift in Gkeyll $(R=1.2 \mathrm{~m})$ and near the location of maximum $E \times B$ drift in GDB $(R=1.24$ $\mathrm{m})$. The fluid and gyrokinetic frequency spectra are comparable in the region where values are greatest, $1-10 \mathrm{kHz}$. The spectra peak at slightly different frequencies, both of which are higher than the experimental peak ${ }^{27}$. The spectrum magnitude for GDB is larger than Gkeyll in the high frequency region. For example, at the highest frequency resolved by Gkeyll $(50 \mathrm{kHz})$, the power was an order of magnitude lower compared to the GDB spectrum. This may be associated with the rapidly-changing small-scale structures observed in GDB, which could alter the spatial and temporal spectra, while only having a minimal effect on turbulent transport.

Finally, we probed statistical properties of the turbulence via the moments of the fluctuations' probability density function. The skewness and the excess kurtosis of the fluctuations as a function of radius are given in figure 21. Concurrent with the agreement in the location of the peak $\delta n_{r m s} /\langle n\rangle_{y, t}$ near 


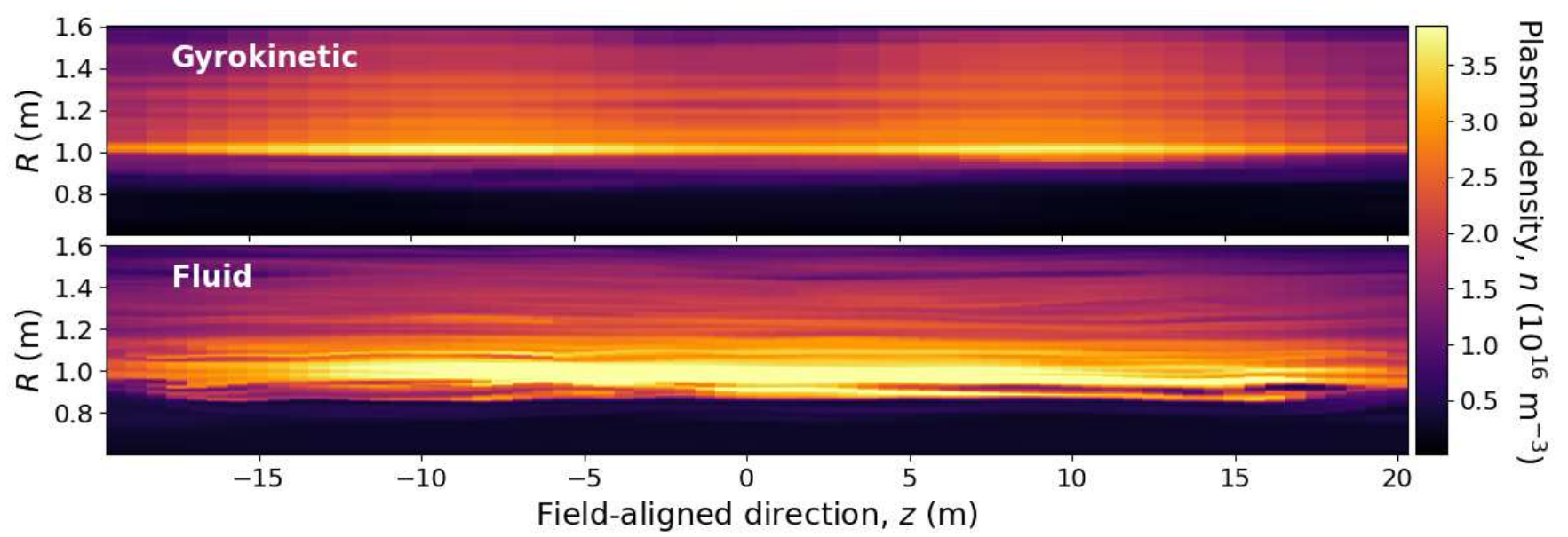

FIG. 19. Gyrokinetic (top) and fluid (bottom) plasma density snapshots at $y=0$ and $t=10 \mathrm{~ms}$. Colors set by extrema in gyrokinetic data.

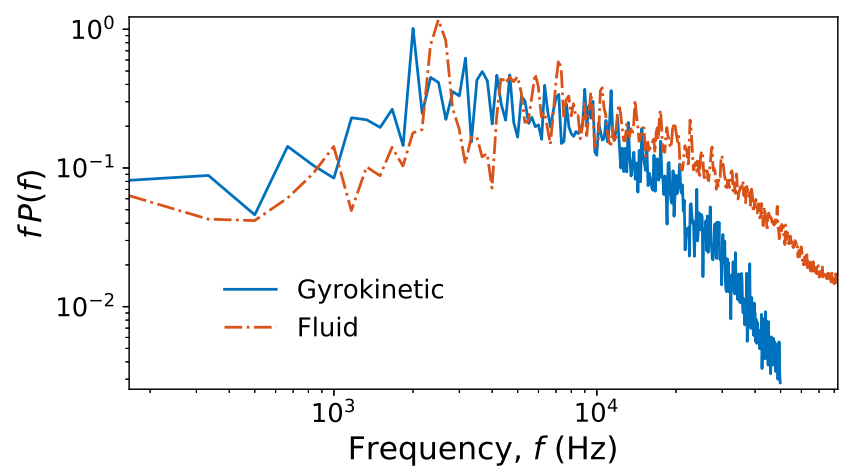

FIG. 20. Frequency spectrum of the density fluctuations of the gyrokinetic (solid blue) and fluid (orange dash-dot) simulations computed near the location of maximum $v_{E}$.

$R=0.9 \mathrm{~m}$ (figure 17), there is also agreement between the fluid and gyrokinetic simulations in the location of maximum skewness and excess kurtosis. There is a second peak in the fluid data at $R \approx 0.76 \mathrm{~m}$ that is absent in the gyrokinetic simulation. Additionally, both skewness and kurtosis were consistently larger on the low-field side in Gkeyll, which corresponds to a flatter density profile in this region and is consistent with previous analyses of intermittent turbulence ${ }^{42}$.

\section{ADDITIONAL DISCUSSION}

In this section we offer further commentary on aspects that are relevant to the physical and numerical facets of comparing GDB and Gkeyll, as well as to potential future comparisons between drift-reduced Braginskii and gyrokinetic models.

The comparison in this manuscript motivated the use of finite parallel heat-flux BCs in the fluid code. Although such BCs were necessary to achieve peak temperatures similar to that in the gyrokinetic simulation, they produced GDB profiles with a high-field side $T_{e}$ that was much smaller than that in Gkeyll. The smallest $T_{e}$ was produced by those finite heat-

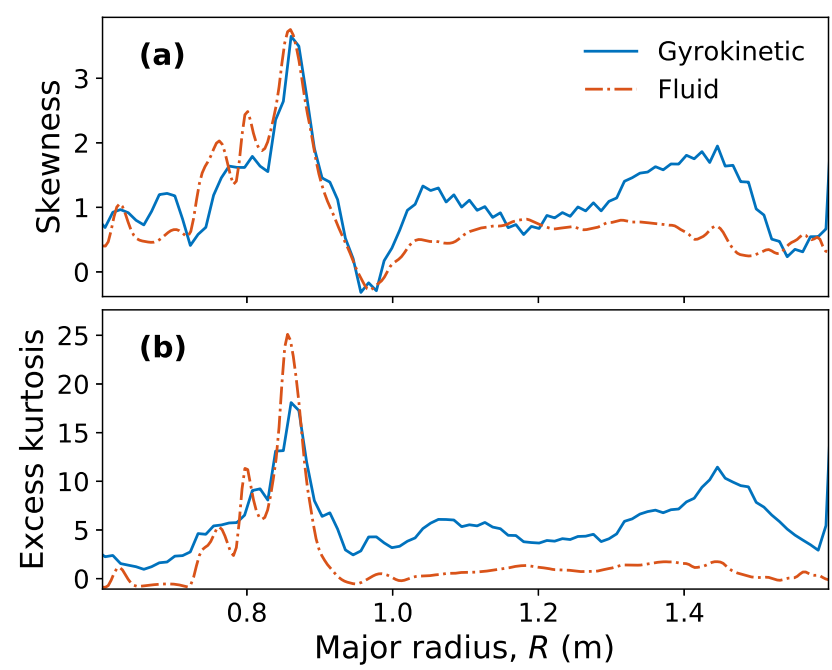

FIG. 21. (a) Skewness and (b) excess kurtosis of the fluctuations as a function of major radius in the gyrokinetic (solid blue) and fluid (orange dash-dot) simulations.

flux BCs not accounting for convective and frictional contributions $\left(q_{\| s} \neq 0\right)$. In the future it may be possible to achieve greater agreement between the high-field side $T_{e}$ produced by the two codes by including the spatial variation of $\kappa_{\|}^{s}$ (supported in GDB but not used here), which would reduce the amount of heat extracted in regions where $T_{e}<T_{e 0}$.

Closing the gap between GDB and Gkeyll could necessitate enhancements to the latter as well. A reader may expect that a Braginskii model, as moments of the kinetic equation, would produce similar results to those obtained by solving the (long wavelength) gyrokinetic equation for this highly collisional plasma. However, aside from the drift-reduction assumed in GDB, the Braginskii equations used here cannot be derived by taking moments of Gkeyll's gyrokinetic equation 1. One conflict is that finite Larmor radius (FLR) effects are only partially incorporated in the Gkeyll model via the Poisson equation (5) but not included in the gyrokinetic 
equation (1). It would be necessary to include FLR effects in order to derive the correct gyroviscous terms from moments of the gyrokinetic equations. Another obstacle is Gkeyll's model collision operator having transport coefficients different to those in the Braginskii model. The viscosity coefficient arising from the Dougherty operator, for example, is $\eta_{0, \text { Dougherty }}^{s}=0.5 n T_{s} /\left(v_{s s}+v_{s r}\right)^{43}$. On the other hand the Braginskii transport coefficients are computed from the exact linearized Landau collision integral, and give the viscosities $\eta_{0}^{i}=0.96 n T_{i} / v_{i i}$ and $\eta_{0}^{e}=0.73 n T_{e} / v_{e i}$. One could change the Braginskii viscosity, and other transport coefficients, to better approximate Gkeyll's. In fact, previous fluid simulations of the Helimak either used an artificial value of $\eta_{0}^{e}$ or neglected it altogether. Including an artificial value of $\eta_{0}^{e}$ is more important when using reduced mass ratios, and it can significantly alter the density and current profiles ${ }^{32}$. However, it is still unclear to what extent matching transport coefficients is crucial to the accurate simulation of this system. A safer course of action is to implement a more accurate collision operator, several of which are being developed that more closely approach Braginskii transport coefficients in the highly collisional limit ${ }^{44-46}$.

The collision operator, through its higher moments, also provides the dissipative channel in the kinetic system. This leads to, for example, collisional drift terms that have been proposed for inclusion in fluid models ${ }^{31}$. These terms are typically not considered as they are thought to be small compared to the artificial diffusion $(\propto \mathcal{D})$ required by the numerical methods used in Braginskii codes. Those diffusion terms can still impact the properties of the turbulence, even though at the levels reported here they did not account for a big portion of GDB's particle balance. In the future exactly conservative formulations of fluid equations that obviate the need for these hyperdiffusion terms may serve as a more reliable approach ${ }^{47}$.

In this sheath-dominated regime, the collisional refinements are likely secondary to the influence of the sheath BCs. As explained in section II, the parallel BCs in both codes are not equivalent. Gkeyll's innovative conducting sheath boundary conditions ${ }^{25}$, have been successfully used to model LAPD, Helimak, and NSTX. Despite their adoption by kinetic codes, the Gkeyll Helimak simulations demonstrate that these BCs do not satisfy the Bohm sheath criterion. On the other hand, fluid codes almost universally impose the Bohm criterion, either $u_{\| i}= \pm c_{s}$ or $\left|u_{\| i}\right|=\geq c_{s}$. It would be useful to know how the kinetic conducting BCs can be modified in order to satisfy the Bohm sheath criterion, perhaps by developing an improved rule for the reflection of the electrons. However, it is unknown whether Bohm sheath BCs are the correct choice for all simulations of laboratory plasmas. Derivation of this condition, for example, assumes ambipolar flows ${ }^{2}$, but a significant fraction of non-ambipolarity has been measured in the tokamak scrape-off-layers ${ }^{48}$. Therefore, more experimental diagnostics at the sheath will likely prove helpful in exploring improved parallel BCs for gyrokinetic and fluid models.

Beyond collision operators and parallel BCs, there are other interesting enhancements that can be pursued with both codes. We previously mentioned that there are better descriptions of
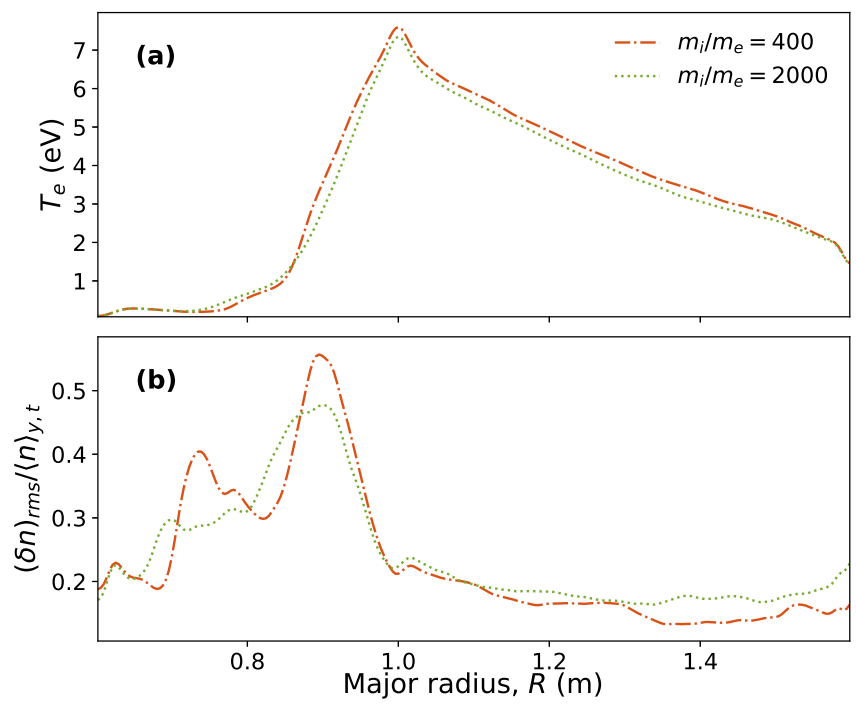

FIG. 22. (a) Radial electron temperature and (b) root-mean-squared (RMS) fluctuation profiles at the bottom sheath for GDB simulations with $m_{i} / m_{e}=400$ (orange dash-dot) and $m_{i} / m_{e}=2000$ (dotted green). These were produced by the same procedure as figures 5 and 17.

the geometry that could be implemented, and new versions of Gkeyll and GDB already contain these capabilities. The new version of Gkeyll, and the flux-coordinate independent approach implemented in GDB tokamak simulations, can be used to incorporate shear and as well as the vertical component of the $E \times B$ velocity. Both codes can also run without the Boussinesq approximation. In tokamak simulations this sophistication did not always alter the results significantly ${ }^{40,49}$, but no exhaustive scans of parameter space have been performed. In the few Helimak simulations we have performed, we note that incorporating the spatial variation $n(x) / B^{2}(x)$ in the ion polarization can add a modest change to the perpendicular profiles and very drastic changes to the parallel current profiles $^{32}$. The aforementioned enhancements may however turn out to be minor when confronted with the high levels of input power radiated away in the Helimak $\left(>90 \%{ }^{27}\right)$; including ionization, radiation cooling of electrons and charge exchange will be essential for fully predictive simulations.

One improvement that we pursued and present here is the use of a higher mass ratio. Since the GDB simulation is more than 10 times cheaper we were able to run it with $m_{i} / m_{e}=$ 2000 with a negligible increase in cost. This is still much less than the true Argon $m_{i} / m_{e}=7.33 \times 10^{4}$. These lighter electrons resulted in a small reduction of the electron temperature profile (figure 22a) and a relatively small correction to the RMS fluctuation profile (figure 22b). Additional results from these simulations can be found in the supplemental materials. 


\section{SUMMARY AND CLOSING REMARKS}

We have presented a detailed comparison of plasma turbulence simulations on open-field lines produced by the fluid code GDB and the (long wavelength) gyrokinetic code Gkeyll. An examination of the radial profiles prompted the implementation of two finite heat-flux BCs $\left(q_{\| s} \neq 0\right.$ and $\left.q_{\| e}^{\text {tot }} \neq 0\right)$ in GDB; these cause important modifications of the temperature profiles. The differences in fluid and gyrokinetic global profiles also motivated the use of larger source amplitudes in GDB than those matching the volume integral of the sources Gkeyll. This was in part necessary due to the lack of particle conservation in GDB, and an examination of this feature highlighted the need to account for missing Jacobian factors and for the inhomogeneity of the magnetic field in the $E \times B$ nonlinearity.

Comparing the gyrokinetic simulation to the $q_{\| s} \neq 0$ fluid simulation with sources increased 2.1875 times and without the $B=B_{(R)}$ accounted for in the $E \times B$ nonlinearity, we observed differences in the high- $f$ spectrum, skewness and kurtosis of density fluctuations, and the density gradients. It was also shown that Gkeyll's conducting sheath BCs do not satisfy the Bohm sheath criterion, while GDB's BCs impose such condition. Sheath BCs can indirectly affect the turbulence and the cross-field spreading of the density profile by altering the particle transit time. At the same time, several other quantities exhibited relative agreement. For example, turbulent structures were qualitatively similar in $x-y$ snapshots, and $E \times B$ flow and turbulent fluctuation profiles were qualitatively, as well as in some cases quantitatively, close.

Future GDB-Gkeyll comparisons will hopefully account for Jacobian factors and inhomogeneities were needed, as well as employ improvements to conservation and a spatially varying collisionality. Ultimately however, it will be crucial to accompany these fluid-kinetic comparisons with experimental validations. If one compares the results presented here with experimental data ${ }^{27}$ it becomes clear that both codes require improvements. The gyrokinetic model produced density fluctuations with a skewness profile and a frequency spectrum that is closer to those observed experimentally than those obtained with the fluid code. But the average density, temperature, and $\delta n_{r m s}$ radial profiles in both codes have notable departures from experimental data. In the future we will use and implement improvements to various features in both codes. This task is partially aided by the reduced cost of GDB simulations; adding and testing new features in the fluid model can help determine which improvements should be prioritized in the gyrokinetic model in order to use resources more efficiently. The new, faster version of Gkeyll also allows for more rapid development and testing.

Finally, a key inquiry of these fluid-kinetic comparisons is whether the fluid or the gyrokinetic model is more appropriate for modeling this and other SOL-like, open-field line systems. The model in Gkeyll may at present be incomplete, but the gyrokinetic system it is developing towards is free of certain limitations inherent to the a moment, collisional closure as Braginskii's. As such it will eventually provide superior accuracy. But both Gkeyll and GDB are not fully developed; given the maturity of these models and the data presented here we cannot provide a universal recommendation on which kind of code is more suitable. In general we can remind the reader that a gyrokinetic code may be better suited to model a lower-collisionality regime and to study kinetic effects. A fluid code is more computationally affordable for modeling a high-collisionality regime and is still able to capture many features of the gyrokinetic simulations. It is clear that improvements are needed in both models, though the general agreement between the two is encouraging for both sides. This work represents a starting point for future comparisons of fluid and kinetic models, including their respective strengths and weaknesses, which will be essential in the effort to achieve predictive modeling of advanced fusion devices.

\section{ACKNOWLEDGMENTS}

We thank Paolo Ricci for his insight into the early simulations of the Texas Helimak and Eric Shi for his role in the development of Gkeyll. Discussion with Ken Gentle and Edward Taylor was also key to understanding the experimental configuration and establishing the numerical setup. We thank Darin Ernst and the rest of the Gkeyll team for their input and helpful suggestions. The simulations presented here carried out at the Texas Advanced Computing Center, the Dartmouth Discovery cluster, and MIT-PSFC's partition of the Engaging cluster at the MGHPCC facility (www.mghpcc.org, funded by DoE grant number DE-FG02-91-ER54109), so we wish to thank the support teams at these facilities for their attention and great work. M.F., B.Z. and B.N.R were supported by the U.S. Department of Energy (DOE) grant DOESC-0010508. M.F. is currently supported by DOE contract DE-FC02-08ER54966. T.N.B. was supported by DOE contract DE-FG02-04ER-54742, through the Institute of Fusion Studies at the University of Texas at Austin, and is currently supported by DOE contract DE-FG02-95ER54309. B.Z. is now supported by the Tokamak Disruption Study SciDAC Project and by DOE contract DE-AC52-07NA27344 through the Lawrence Livermore National Laboratory. A.H. and G.W.H. are supported by the High-Fidelity Boundary Plasma Simulation SciDAC Project, part of the DOE Scientific Discovery Through Advanced Computing (SciDAC) program, through DOE contract DE-AC02-09CH11466 for the Princeton Plasma Physics Laboratory. A.H. is also supported by the Air Force Office of Scientific Research under contract FA9550-15-1-0193. The reduced data used to produce the figures in this work is available upon request, and input files for the simulations can also be furnished should the raw data need regeneration.

\section{Appendix A: GDB normalization}

The two-fluid equations 7-15 are solved in the following normalized form:

$$
\frac{d^{e} \ln n}{d t}=-\nabla \cdot \boldsymbol{v}_{\perp}-\varepsilon_{v} \nabla_{\|} u_{\| e}+\frac{S_{n}}{n}+\mathcal{D}_{\ln n}
$$




$$
\begin{aligned}
\frac{\partial \omega}{\partial t}= & \mathcal{C}_{\left(p_{e}\right)}+\tau \mathcal{C}_{\left(p_{i}\right)}+\frac{\varepsilon_{v}}{\alpha_{d} \varepsilon_{R}} \nabla_{\|} j_{\|}-\mathcal{C}_{\left(G_{i}\right)} \\
& -\nabla \cdot\left\{\frac{n}{B^{3}}[\phi, h]+\sqrt{\tau} \varepsilon_{v} \frac{n}{B^{2}} u_{\| i} \nabla_{\|} \boldsymbol{h}\right\}+\mathcal{D}_{\omega} \\
\frac{d^{e} u_{\| e}}{d t}= & \frac{m_{i}}{m_{e}} \varepsilon_{v}\left(\frac{\nabla_{\|} \phi}{\alpha_{d}}-\frac{\nabla_{\|} p_{e}}{n}-0.71 \nabla_{\|} T_{e}+4 \frac{\nabla_{\|} G_{e}}{n}\right) \\
& +\eta j_{\|}+\varepsilon_{R} \alpha_{d} T_{e} \mathcal{C}_{\left(u_{\| e}\right)}-\frac{u_{\| e}}{n} S_{n}+\mathcal{D}_{u_{\| e}},
\end{aligned}
$$$$
\frac{d^{i} u_{\| i}}{d t}=-\frac{\varepsilon_{v}}{\sqrt{\tau}}\left(\frac{\nabla_{\|} \phi}{\alpha_{d}}+\tau \frac{\nabla_{\|} p_{i}}{n}-0.71 \nabla_{\|} T_{e}-4 \frac{\nabla_{\|} G_{i}}{n}\right)
$$$$
-\frac{m_{e}}{m_{i}} \frac{\eta}{\sqrt{\tau}} j_{\|}-\varepsilon_{R} \tau \alpha_{d} T_{i} \mathcal{C}_{\left(u_{\| i}\right)}-\frac{u_{\| i}}{n} S_{n}+\mathcal{D}_{u_{\| i}}
$$

$$
\begin{aligned}
\frac{d^{e} \ln T_{e}}{d t}= & \frac{5}{3} \varepsilon_{R} \alpha_{d} \mathcal{C}_{\left(T_{e}\right)}+\frac{1}{p_{e}} \nabla_{\|} \kappa^{e} \nabla_{\|} T_{e}+\frac{2}{3}\left[-\nabla \cdot \boldsymbol{v}_{\perp}\right. \\
& -\varepsilon_{v} \nabla_{\|} u_{\| e}+\frac{0.71 \varepsilon_{v}}{n}\left(\nabla_{\|} j_{\|}-j_{\|} \nabla_{\|} \ln T_{e}\right) \\
& \left.+\frac{S_{E, e}}{p_{e}}-\frac{3}{2} \frac{S_{n}}{n}\right]+\mathcal{D}_{\ln T_{e}}
\end{aligned}
$$$$
\frac{d^{i} \ln T_{i}}{d t}=-\frac{5}{3} \tau \varepsilon_{R} \alpha_{d} \mathcal{C}_{\left(T_{i}\right)}+\frac{1}{p_{i}} \nabla_{\|} \kappa^{i} \nabla_{\|} T_{i}+\frac{2}{3}\left(-\nabla \cdot v_{\perp}\right.
$$$$
\left.-\sqrt{\tau} \varepsilon_{v} \nabla_{\|} u_{\| i}+\varepsilon_{v} \frac{\nabla_{\|} j_{\|}}{n}+\frac{S_{E, i}}{p_{i}}-\frac{3}{2} \frac{S_{n}}{n}\right)+\mathcal{D}_{\ln T_{i}}
$$

where we used $\nabla \cdot \boldsymbol{v}_{\perp}=\varepsilon_{R}\left[\mathcal{C}_{(\phi)}-\alpha_{d} \mathcal{C}_{\left(p_{e}\right)} / n\right], \boldsymbol{h}=\nabla \phi+$ $\tau \alpha_{d}\left(\nabla p_{i}\right) / n, \omega=\nabla \cdot n \boldsymbol{h} / B^{2}$ is the generalized vorticity, and $j_{\|}=n\left(\sqrt{\tau} u_{\| i}-u_{\| e}\right)$ is the normalized current. The dynamic variables in physical units can be retrieved as follows: $\left.n_{\text {phys }}=n_{\text {ref }} n, T_{\alpha, \text { phys }}=T_{\alpha, \text { ref }} T_{\alpha}, \phi_{\text {phys }}=B_{\text {ref }} a^{2} \phi /\left(c t_{\text {ref }}\right)\right)$, $v_{\| \alpha, \text { phys }}=v_{\| \alpha, \text {,ref }} v_{\| \alpha}, \quad j_{\| \text {phys }}=e n_{\text {ref }} u_{\| e, \text { ref }} j_{\|} . \quad$ The dimensionless magnetic field and major radius are defined by $B_{\text {phys }}=B_{\text {ref }} B$ and $R_{\text {phys }}=R_{\text {ref }} R$. The reference values were $n_{\text {ref }}=n_{e 0}, B_{\text {ref }}=B_{0}, R_{\text {ref }}=R_{0}, T_{s, \text { ref }}=T_{s 0}, u_{\| s, \text { ref }}=$ $c_{s s, \text { ref }}=\sqrt{T_{s, \text { ref }} / m_{s}}$. Perpendicular lengths are normalized to the machine's width $a=1 \mathrm{~m}$, and parallel ones to the plasma center major radius $R_{0}$. The reference time is the interchange-like timescale $t_{\text {ref }}=\sqrt{a R_{0} / 2} / c_{s e \text {,ref }}$, which for the reference parameters in this work gives $t_{\text {ref }}=1.5145 \times 10^{-4} \mathrm{~s}$. The normalized transport coefficients are $\varepsilon_{G_{i}}=0.08 \tau \tau_{i, \text { ref }} / t_{\text {ref }}, \varepsilon_{G_{e}}=0.73 \tau_{e, \text { ref }} /\left(12 t_{\text {ref }}\right), \eta=$ $0.51 t_{\text {ref }} / \tau_{e, \text { ref }}, \kappa^{e}=3.2\left[2 t_{\text {ref }} /\left(3 n_{\text {ref }} L_{\| \text {ref }}^{2}\right)\right] \tau_{e, \text { ref }} n_{\text {ref }} T_{e, \text { ref }} / m_{e}$ and $\kappa^{i}=3.9\left[2 t_{\text {ref }} /\left(3 n_{\text {ref }} L_{\| \text {ref }}^{2}\right)\right] \tau_{i \text {,ref }} n_{\text {ref }} T_{i, \text { ref }} / m_{i}$. Here $\tau_{s, \text { ref }}$ refers to the collisional period ${ }^{33}$. We also employ the dimensionless variables $\varepsilon_{R}=2 a / R_{0}, \quad \varepsilon_{v}=c_{s e \text {,ref }} t_{\text {ref }} / R_{0}, \quad \alpha_{d}=$ $c_{s e \text {,ref }}^{2} t_{\text {ref }} /\left(\Omega_{i, \text { ref }} a^{2}\right)$. The normalized functions arising from the gyroviscous stress tensor are now

$$
\begin{aligned}
& G_{i}=\varepsilon_{G_{i}}\left[4 \sqrt{\tau} \varepsilon_{v} \nabla_{\|} u_{\| i}+\varepsilon_{R}\left(\mathcal{C}_{(\phi)}+\tau \alpha_{d} \frac{\mathcal{C}_{\left(p_{i}\right)}}{n}\right)\right], \\
& G_{e}=\varepsilon_{G_{e}}\left[4 \varepsilon_{v} \nabla_{\|} u_{\| e}+\varepsilon_{R}\left(\mathcal{C}_{(\phi)}-\alpha_{d} \frac{\mathcal{C}_{\left(p_{e}\right)}}{n}\right)\right] .
\end{aligned}
$$

The normalized form of the time rate of change is

$$
\begin{aligned}
& \frac{d^{e} F}{d t}=\frac{\partial F}{\partial t}+\frac{1}{B}[\phi, F]+\varepsilon_{v} u_{\| e} \nabla_{\|} F, \\
& \frac{d^{i} G}{d t}=\frac{\partial G}{\partial t}+\frac{1}{B}[\phi, G]+\sqrt{\tau} \varepsilon_{v} u_{\| i} \nabla_{\|} G,
\end{aligned}
$$

and the normalized curvature operator is $\mathcal{C}_{(F)}=\partial F / \partial y$. We also require the appropriate normalized form of the finite heat flux BCs in equation 18. This is

$$
\begin{aligned}
& \nabla_{\|} \ln T_{e}=\mp \frac{\sqrt{\varepsilon_{R}}}{3 \kappa^{e}} \gamma_{e} n \sqrt{T_{e}+\tau T_{i}} \exp \left[\Lambda-\max \left(\frac{\phi}{\alpha_{d} T_{e}}, 0\right)\right], \\
& \nabla_{\|} \ln T_{i}=\mp \frac{\sqrt{\varepsilon_{R}}}{3 \kappa^{i}} \gamma_{i} n \sqrt{T_{e}+\tau T_{i}},
\end{aligned}
$$

where the upper (lower) sign is used at the top (bottom) sheath. The heat transmission coefficients are $\gamma_{i}=2.5 \tau T_{i} / T_{e}$ and $\gamma_{e}=2+\left|\phi /\left(\alpha_{d} T_{e}\right)\right|$. On the other hand, the finite electron heat-flux $\mathrm{BC}$ that takes into account the convective and frictional component of the heat-flux (equation 19) is implemented as

$$
\begin{aligned}
\nabla_{\|} & \ln T_{e}=\mp n \sqrt{T_{e}+\tau T_{i}}\{0.71 \\
& \left.+\frac{\sqrt{\varepsilon_{R}}}{3 \kappa^{e}}\left(\gamma_{e}-\frac{5}{2}-0.71\right) \exp \left[\Lambda-\max \left(\frac{\phi}{\alpha_{d} T_{e}}, 0\right)\right]\right\} .
\end{aligned}
$$

Finally, unless stated otherwise the normalized diffusion coefficients were

\begin{tabular}{c|c|c|c} 
& $\chi_{x}\left(10^{-17}\right)$ & $\chi_{y}\left(10^{-18}\right)$ & $\chi_{\|}$ \\
\hline $\mathcal{D}_{\ln n}$ & 0.30686 & 0.34384 & 0.30572 \\
$\mathcal{D}_{\ln T_{e}}$ & 0.30686 & 0.34384 & 0 \\
$\mathcal{D}_{\ln T_{i}}$ & 0.30686 & 0.34384 & 0 \\
$\mathcal{D}_{u_{\| i}}$ & 0.30686 & 0.34384 & 0.030572 \\
$\mathcal{D}_{\omega}$ & 6.5770 & 7.3697 & 0.30572 \\
$\mathcal{D}_{u_{\| e}}$ & 1.4206 & 1.5919 & 0.30572.
\end{tabular}

${ }^{1}$ M. Kotschenreuther, W. Dorland, Q. P. Liu, G. W. Hammett, M. A. Beer, S. A. Smith, A. Bondeson, and S. C. Cowley, "First principles calculations of tokamak energy transport," in Proceedings of the 16th International Conference on Fusion Energy (Montreal, 1996) pp. 371-383.

${ }^{2}$ P. Stangeby, The Plasma Boundary of Magnetic Fusion Devices, Series in Plasma Physics and Fluid Dynamics (Taylor \& Francis, 2000).

${ }^{3}$ J. R. Myra, D. A. D'Ippolito, D. P. Stotler, S. J. Zweben, B. P. Leblanc, J. E. Menard, R. J. Maqueda, and J. Boedo, "Blob birth and transport in the tokamak edge plasma: Analysis of imaging data," Phys. Plasmas2 13, 092509 (6).

${ }^{4}$ R. A. Pitts, P. Andrew, G. Arnoux, T. Eich, W. Fundamenski, A. Huber, C. Silva, D. Tskhakaya, and J. E. Contributors, "ELM transport in the JET scrape-off layer," Nucl. Fusion 47, 1437-1448 (2007).

${ }^{5}$ National Academies of Sciences, Engineering, and Medicine, Final Report of the Committee on a Strategic Plan for U.S. Burning Plasma Research (The National Academies Press, Washington, DC, 2019).

${ }^{6}$ P. Ricci, B. N. Rogers, and S. Brunner, "High- and low-confinement modes in simple magnetized toroidal plasmas," Phys. Rev. Lett. 100, 6-9 (2008). ${ }^{7}$ B. Li, B. N. Rogers, P. Ricci, and K. W. Gentle, "Plasma transport and turbulence in the helimak: Simulation and experiment," Physics of Plasmas 16, 082510 (2009).

${ }^{8} \mathrm{P}$. Ricci and B. N. Rogers, "Turbulence phase space in simple magnetized toroidal plasmas," Phys. Rev. Lett. 104, 1-4 (2010). 
${ }^{9}$ B. Li, B. N. Rogers, P. Ricci, K. W. Gentle, and A. Bhattacharjee, "Turbulence and bias-induced flows in simple magnetized toroidal plasmas," Phys. Rev. E 83, 056406 (2011).

${ }^{10}$ P. Tamain, H. Bufferand, G. Ciraolo, C. Colin, D. Galassi, P. Ghendrih, F. Schwander, and E. Serre, "The TOKAM3X code for edge turbulence fluid simulations of tokamak plasmas in versatile magnetic geometries," J. Comput. Phys. 321, 1-36 (2016).

${ }^{11}$ F. Halpern, P. Ricci, S. Jolliet, J. Loizu, J. Morales, A. Mosetto, F. Musil, F. Riva, T. Tran, and C. Wersal, "The GBS code for tokamak scrape-off layer simulations,” J. Comput. Phys. 315, 388-408 (2016).

${ }^{12}$ A. Stegmeir, D. Coster, A. Ross, O. Maj, K. Lackner, and E. Poli, "GRILLIX: A 3D turbulence code based on the flux-coordinate independent approach," Plasma Phys. Control. Fusion 60 (2018), 10.1088/1361-6587/aaa373.

${ }^{13}$ B. D. Dudson, M. V. Umansky, X. Q. Xu, P. B. Snyder, and H. R. Wilson, "BOUT++: A framework for parallel plasma fluid simulations," Comput. Phys. Commun. 180, 1467-1480 (2009).

${ }^{14}$ B. Zhu, M. Francisquez, and B. N. Rogers, "GDB: A global 3D twofluid model of plasma turbulence and transport in the tokamak edge," Computer Physics Communications 232, 46 - 58 (2018).

${ }^{15}$ F. D. Halpern, J. L. Terry, S. J. Zweben, B. LaBombard, M. Podesta, and P. Ricci, "Comparison of 3D flux-driven scrape-off layer turbulence simulations with gas-puff imaging of Alcator C-Mod inner-wall limited discharges," Plasma Phys. Control. Fusion 57, 054005 (2015).

${ }^{16}$ B. Chen, X. Xu, T. Xia, M. Porkolab, E. Edlund, B. LaBombard, J. Terry, J. Hughes, S. Mao, M. Ye, and Y. Wan, "Edge turbulence and divertor heat flux width simulations of Alcator C-Mod discharges using an electromagnetic two-fluid model," Nuclear Fusion 57, 116025 (2017).

${ }^{17}$ R. E. Waltz, F. D. Halpern, Z. Deng, and J. Candy, "Kinetic fluid moments closure for a magnetized plasma with collisions," (2019), arXiv: 1901.02429

${ }^{18} \mathrm{~J} . \mathrm{Ng}$, Fluid closures for the modeling of reconnection and instabilities in magnetotail current sheets, Ph.D. thesis, Princeton University (2018).

${ }^{19}$ C. S. Chang, S. Ku, A. Loarte, V. Parail, F. Köchl, M. Romanelli, R. Maingi, J.-W. Ahn, T. Gray, J. Hughes, B. LaBombard, T. Leonard, M. Makowski, and J. Terry, "Gyrokinetic projection of the divertor heat-flux width from present tokamaks to ITER,” Nucl. Fusion 57, 116023 (2017).

${ }^{20}$ Q. Pan, D. Told, E. Shi, G. W. Hammett, and F. Jenko, "Full- $f$ version of GENE for turbulence in open-field-line systems," Phys. Plasmas 25, 1-11 (2018).

${ }^{21}$ V. Grandgirard, J. Abiteboul, J. Bigot, T. Cartier-michaud, and N. Crouseilles, "A 5D gyrokinetic full- $f$ global semiLagrangian code for flux-driven ion turbulence simulations," Comput. Phys. Commun. 207, 35-68 (2016).

${ }^{22}$ L. Chôné, T. P. Kiviniemi, S. Leerink, P. Niskala, and R. Rochford, "Improved boundary condition for full- $f$ gyrokinetic simulations of circular-limited tokamak plasmas in ELMFIRE," Contrib. to Plasma Phys. 58, 534-539 (2018).

${ }^{23}$ M. H. Boesl, A. Bergmann, A. Bottino, D. Coster, E. Lanti, N. Ohana, and F. Jenko, "Gyrokinetic full- $f$ particle-in-cell simulations on open field lines with PICLS," (2019), arXiv:1908.00318.

${ }^{24}$ M. A. Dorf, M. R. Dorr, J. A. Hittinger, R. H. Cohen, and T. D. Rognlien, "Continuum kinetic modeling of the tokamak plasma edge," Phys. Plasmas 23, 056102 (2016).

${ }^{25}$ E. L. Shi, G. W. Hammett, T. Stoltzfus-Dueck, and A. Hakim, "Gyrokinetic continuum simulation of turbulence in a straight open-field-line plasma," Journal of Plasma Physics 83, 905830304 (2017).

${ }^{26}$ E. L. Shi, G. W. Hammett, T. Stoltzfus-Dueck, and A. Hakim, "Full- $f$ gyrokinetic simulation of turbulence in a helical open-field-line plasma," Physics of Plasmas 26, 012307 (2019).

${ }^{27}$ T. N. Bernard, E. L. Shi, K. W. Gentle, A. Hakim, G. W. Hammett, T. Stoltzfus-Dueck, and E. I. Taylor, "Gyrokinetic con- tinuum simulations of plasma turbulence in the texas helimak," Physics of Plasmas 26, 042301 (2019).

${ }^{28}$ E. L. Shi, Gyrokinetic continuum simulation of turbulence in open-fieldline plasmas, Ph.D. thesis, Princeton University (2017).

${ }^{29}$ A. Hakim, G. Hammett, E. Shi, and N. Mandell, "Discontinuous galerkin schemes for a class of hamiltonian evolution equations with applications to plasma fluid and kinetic problems," (2019), arXiv:1908.01814.

${ }^{30}$ A. Geraldini, F. I. Parra, and F. Militello, "Gyrokinetic treatment of a grazing angle magnetic presheath," Plasma Physics and Controlled Fusion 59, 025015 (2017).

${ }^{31}$ J. Madsen, V. Naulin, A. H. Nielsen, and J. J. Rasmussen, "Collisional transport across the magnetic field in drift-fluid models," Phys. Plasmas 23 (2016), 10.1063/1.4943199.

${ }^{32}$ M. Francisquez, Global Braginskii modeling of magnetically confined boundary plasmas, Ph.D. thesis, Dartmouth College, Hanover, NH 03755 (2018).

${ }^{33}$ J. D. Huba, Plasma Physics (Naval Research Laboratory, Washington, DC, 2013) pp. 1-71.

${ }^{34}$ M. Francisquez, B. Zhu, and B. N. Rogers, "Multigrid treatment of implicit continuum diffusion," Comput. Phys. Commun. 236, 104-117 (2019).

${ }^{35}$ J. Loizu, P. Ricci, F. D. Halpern, and S. Jolliet, "Boundary conditions for plasma fluid models at the magnetic presheath entrance," Physics of Plasmas 19, 122307 (2012), https://doi.org/10.1063/1.4771573.

${ }^{36}$ A. Stegmeir, A. Ross, T. Body, M. Francisquez, W. Zholobenko, D. Coster, O. Maj, P. Manz, F. Jenko, B. N. Rogers, and K. S. Kang, "Global turbulence simulations of the tokamak edge region with GRILLIX," Phys. Plasmas 26 (2019), 10.1063/1.5089864.

${ }^{37}$ S. A. Smith and G. W. Hammett, "Eddy viscosity and hyperviscosity in spectral simulations of 2D drift wave turbulence," Phys. Plasmas 4, 978 (1997).

${ }^{38}$ T. Xia, X. Xu, and P. Xi, "Six-field two-fluid simulations of peeling-ballooning modes using BOUT++," Nuclear Fusion 53, 073009 (2013).

${ }^{39}$ F. Halpern, P. Ricci, S. Jolliet, J. Loizu, and A. Mosetto, "Theory of the scrape-off layer width in inner-wall limited tokamak plasmas," Nuclear Fusion 54, 043003 (2014).

${ }^{40}$ M. Francisquez, B. Zhu, and B. Rogers, "Global 3D Braginskii simulations of the tokamak edge region of IWL discharges," Nuclear Fusion 57, 116049 (2017).

${ }^{41}$ B. D. Dudson and J. Leddy, "Hermes: global plasma edge fluid turbulence simulations," Plasma Physics and Controlled Fusion 59, 054010 (2017).

${ }^{42}$ D. D'Ippolito, J. Myra, and S. Zweben, "Convective transport by intermittent blob-filaments: Comparison of theory and experiment," Phys. Plasmas 18, 060501 (2011).

${ }^{43} \mathrm{M}$. W. Anderson and T. M. O'Neil, "Eigenfunctions and eigenvalues of the Dougherty collision operator," Phys. Plasmas 14, 052103 (2007).

${ }^{44}$ Q. Pan and D. R. Ernst, "Gyrokinetic Landau collision operator in conservative form,” Phys. Rev. E 99, 023201 (2019).

${ }^{45}$ H. Sugama, S. Matsuoka, S. Satake, M. Nunami, and T. Watanabe, "Improved linearized model collision operator for the highly collisional regime," (2019), arXiv:1906.07427.

${ }^{46}$ R. Jorge, B. J. Frei, and P. Ricci, "Non-Linear Gyrokinetic Coulomb Collision Operator," (2019), arXiv:1906.03252.

${ }^{47}$ F. D. Halpern and R. E. Waltz, "Anti-symmetric plasma moment equations with conservative discrete counterparts," Physics of Plasmas 25, 060703 (2018).

${ }^{48}$ R. Dejarnac, P. Stangeby, R. Goldston, E. Gauthier, J. Horacek, M. Hron, M. Kocan, M. Komm, R. Panek, R. Pitts, and P. Vondracek, "Understanding narrow sol power flux component in compass limiter plasmas by use of langmuir probes," Journal of Nuclear Materials 463, 381 - 384 (2015).

${ }^{49}$ A. Ross, Extension of GRILLIX: Towards a global fluid turbulence code for realistic magnetic geometries, Ph.D. thesis, Technische Universität München (2018). 